\title{
An Environment for Automated Measurement of Energy Consumed by Mobile and Embedded ComputingDevices
}

\author{
Armen Dzhagaryan, ${ }^{1}$ Aleksandar Milenković, ${ }^{1}$ Mladen Milosevic, ${ }^{2}$ Emil Jovanov ${ }^{1}$ \\ ${ }^{1}$ Department of Electrical and Computer Engineering \\ The University of Alabama in Huntsville \\ 301 Sparkman Drive \\ Huntsville, AL, 35899 U.S.A. \\ \{aad0002, milenka,emil.jovanov\}@uah.edu \\ ${ }^{2}$ Philips Research North America \\ 2 Canal Park, $3^{\text {rd }}$ Floor \\ Cambridge, MA 02141, USA \\ mladem@gmail.com
}

\begin{abstract}
Mobile and embedded computing devices have become the dominant type of computing platforms. Energy-efficiency is a key requirement for these devices, underscored by growing reliance of consumers on services delivered through them and their growing complexity and sophistication. A detailed measurementbased characterization of energy consumedby applications running on mobile and embedded computing devices is important for both device manufacturers and application developers, as it may identify energydemanding components and activities and guide optimizations. In this paper, we describe an environment for automated energy measurements of applications running on Android mobile and bare embedded computing devices. We discuss hardware and software aspects of the environment and several approaches to runtime capturing and timestamping of activities of interest. Finally, we demonstrate the use of the environment in several case studies conducted on two smartphones and a wearable device.
\end{abstract}

Keywords-Mobile computing; Computerized instrumentation; Current measurement; Energy measurement; Performance evaluation

\section{INTRODUCTION}

Battery-powered computing devices such as mobile devices (smartphones, tablets, e-readers) and wearable consumer devices (e.g., fitness trackers, smart watches, headsets, glasses, smart clothing, wearable cameras) have become the dominant computing platformsfor generating and consuming digital information. The number of smartphones and tablets shipped in 2015 reached 1.43 billion and 241 million, respectively, whereas the number of personal computers was 290 million[1], [2]. Modern smartphones and tablets have 
evolved into powerful computing platforms with significant processing power, storage capacity, myriad of communication interfaces, and numerous sensors. New applications have emerged in areas of communication, navigation, social networking, mobile health, and entertainment. The number of wearabledevices shipped in 2015 was 232 million, including 50.4 million smartwatches, 128.5 million Bluetooth headsets, and $\sim 35$ million wristbands. Modern wearable devices are rapidly proliferating, offering new applications across the health, fitness, sports, and communication segments. They have a potential not only to empower consumers but also to transform modern business processes and improve worker productivity.

Both mobile and wearable devices employ batteries as the source of energy and share common requirements to be small and lightweight and to provide a rich set of functions. However, battery capacity is directly proportional to its size and weight. Growing dependency of users on services delivered through their battery-powered devices makes their energy-efficient operation a top priority. Energy efficiency is a prime design requirement for device manufacturers and application developers alike. It is driven by several key factors, including (i) limited energy capacity of batteries, (ii) cost considerations favoring less expensive packaging, and (iii) user convenience favoring lightweight designs with small form factors that operate for long periods without battery recharges.

A number of recent research studies have focused on power profiling and power estimation of mobile computing platforms. Carroll and Heiser quantified energy consumption of each component in a mobile device by performing rigorous tests and then simulating a number of usage scenarios on mobile devices [3]. Rice and Hay profiled the energy consumption of connecting and transmitting data over a wireless network [4], [5]. Bircher and John used processor performance counters and system-specific models to estimate consumption of CPU, memory, disk, and I/O [6]. Pathak et al. [7], [8] and Li and John [9] used system call tracing and known observations of the system to generate models that can perform run-time power estimation with fine-grained measurements. 
Runtime power measurements on real mobile devices running common software platforms such as Android, iOS, Tizen, or Windows Phone are important for both researchers and mobile application developers. Measurement frameworks can capture complex interactions between hardware and software stacks that become more and more sophisticated with introduction of systems-on-a-chip (SoCs) with multiple processor cores and a number of customized hardware accelerators. Measurements on real devices can help research studies that target power optimizations or those that target developing analytical models for energy estimation based on parameters derived from real platforms. For mobile developers, adding a power perspective to application debugging and testing may guide optimizations that will result in more energyefficient mobile applications.Runtime power measurements can be even more critical for wearable devices because they may offer developers deeper insights into energy requirements of both hardware and software components. Thus, system developers may select more energy efficient hardware components and application developersmay better exploit energy-efficient operating modes and perform trade-offs between computation and communication tasks.

Whereas several prior studies focused on capturing power traces on smartphones [3]-[5], [10] and wireless sensor network platforms [11], they relied on manual control and post-processing for synchronization of power traces with events in profiled programs or focused on early smartphones and software platforms. In addition, they relied on hardware setups that required inserting a shunt resistor on the power supply line, thus introducing a slight deviation in the power supply of the device under test.

In this paper, we introduce an environment for automated power and energy measurements of modern mobile and wearable devices. Our hardware setup includesa device under test, a National Instruments' (NI)chassis with a battery simulator and adata acquisition device (DAQ), and a workstation. Our custom program running on the workstation interfaces both the device under test and theNI chassis. The program provides (i) a number of configuration options to customize the energy profiling, (ii) functions to remotely controlapplications and activities executed on the device under test, (iii) functions to synchronize running 
applications with collecting current samples from the battery simulator, and (iv) scripts for calculating the energy consumed. We describe several approaches to capturing timestamps on devices under test that delimit the profiled activities. The first approach for mobile devices relies on the native Android logging system and does not require any changes in applications that are being profiled. The second approach also relies on the native Android logging system and custom messages inserted in the source code by developers. The third approach relies on CyanogenMod Android and common Linux-like utilities to support launching and timestamping of applications.In addition, we describe an approach for capturing hardware timestamps on bare-metal embeddedplatforms (platforms that do not have operating system)to synchronize collection ofcurrent samples with profiled applications.

Some of the key advantages of the proposed measuring setup are as follows.

- No hardware modifications to mobile devices. The setup requires no hardware modifications or instrumentation of mobile and wearable devices; the device's battery is simply replaced with probes coming from the battery simulator.

- Automated test execution. The measurements are fully automated and controlled by scripts prepared in advance and thus do not require interactive user participation. The scripts can control energy profiling of a number of applications profiled in a single test run.

- Automated synchronization. The workstation and the device under test are time-synchronized using standard network synchronization protocols, thus allowing for precise timestamping of activities of interest. The workstation and bare-metal embedded platforms could optionally be synchronized though the DAQ and lightweight instrumentation of profiled applications.

- High resolution and accuracy. The setup allows collection of up to 200,000 samples per second of power supply current with an accuracy of $1 \mu \mathrm{A}$, thus providing a deep insight into inner operations of internal components. 
The rest of this paper is organized as follows. Section II describes the hardware and software aspects of the setup for energy measurement. Section III describes approaches to profiling Android applications,including different methods for collecting timestamps that delimit the activities of interestin time. Section IV describes an approach to profiling applications running on bare-metal embedded platforms. Section V demonstrates the use of the setup in estimating energy-efficiency of several important activities. Section VI surveys related work, and Section VII concludes the paper.

\section{Measuring Setup}

Our setup for energy profiling, shown in Figure 1, consists of a device under test, an NI PXIe-4154 battery simulator[12], an NI PXIe-6361data acquisition (DAQ) with an external Shielded I/O ConnectionBlock (SCB-68), and a workstation. Figure 2 shows a block diagram of the setupwith main components and communications between them. The block diagram illustrates two types of devices under test: an Android mobile and a bare-metal embedded device.As examples of mobile devices we use a Google's Nexus 4 smartphone [13] running Android 4.3.2 operating system [14] and an OnePlus One smartphone [15] running CyanogenMod 12.1 [16]. As an example of an embedded devicewe usea custom wearable platform calledSmart Button[17] that is developed for automated assessment of mobility in elderly and Parkinson's patients. Whereas the paper focuses on energy profiling of Android platforms and a bare-metal embedded platforms, our hardware setup can be used to profile applications running on other mobile software platforms such as iOS, Tizen, or Windows Phone, as well as on other types of embedded platforms.

The battery simulator and the data acquisition device are connected to an MXI-Express Interface card inside the workstation. The battery simulator is used (i) to power a device under test through probes, thus bypassing the actual battery of the profiled device, and (ii) to measure the current drawn by the device while running applications. The DAQ is used to assert digital outputs or monitor digital inputs that can be connected to the device under test and used to trigger or observe events of interest for power profiling. The workstation runs our custom program called mLViewPowerProfile that interfaces (i) the device under test to 
manage activities and applications that are being profiled, (ii) the battery simulator to capture and record the current sample measurements, and (iii) the DAQ to capture hardware events triggered by software running on embedded platforms. The following subsections shed more light on each component in our setup.

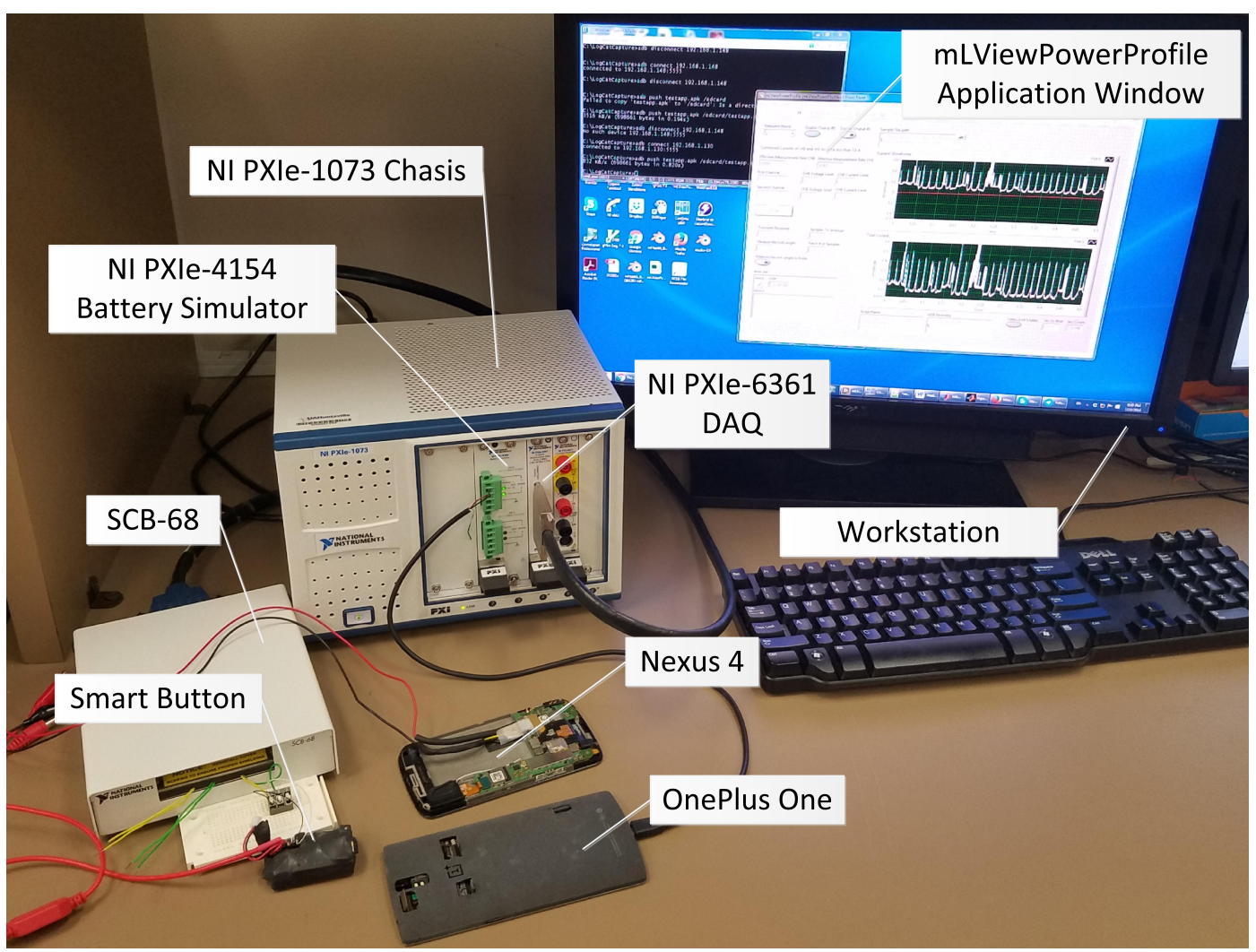

Figure 1. Hardware setup for energy profiling including devices under test:Nexus 4, OnePlus One, and Smart Button 


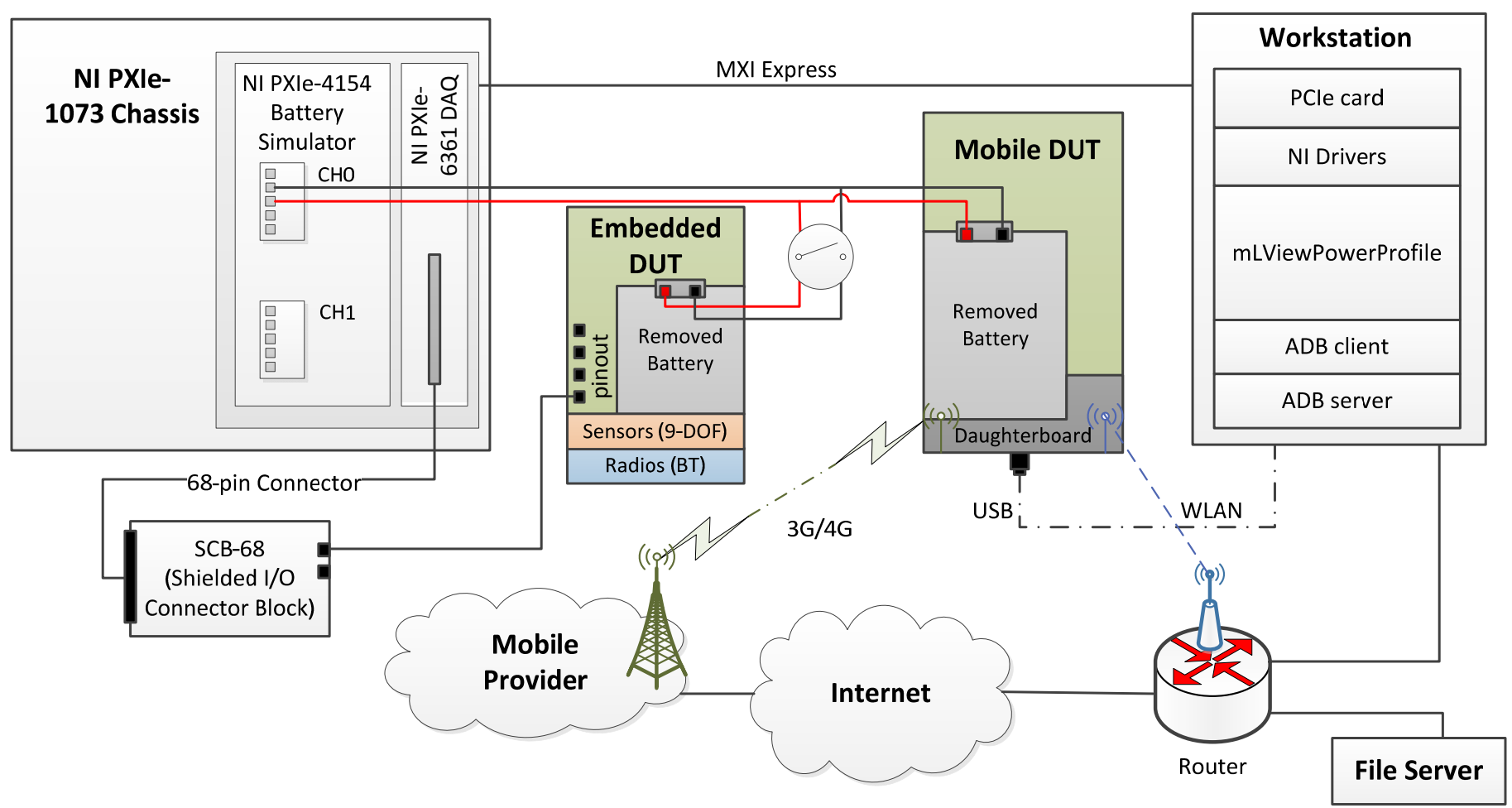

Figure 2. Block diagram of the hardware setup for energy profiling for two types of devices under test: a mobile computing device and a bare-metal embedded device

\section{A. Devices under Test}

The Google's Nexus 4 smartphone [17] is powered by a Qualcomm's Snapdragon S4 Pro (APQ8064) SoC[18] that includes a quad-core ARM processor running at up to $1.512 \mathrm{GHz}$ clock and an Adreno 320 graphics processor [19]. Nexus 4 has 2 GB of RAM memory and 16 GB of built-in internal storage. It uses a 4.7 inch display, and includes a 1.3 megapixel front-facing camera and an 8 megapixel rear-facing camera. It supports a range of connectivity options including WLAN 802.11n, Bluetooth 4.0, USB, HDMI, and several cellular network protocols such as GSM/EDGE/GPRS, 3G UMTS/HSPA+/DC-HSP+, and HSDPA+.Nexus 4 runs Android version 4.3.2 (Jelly Bean). In some cases, an upgrade to Android may be beneficial to (i) support applications and setups not readily available on native Android, and (ii) to further automate performance and energy measurements. Our alternative smartphone setup requires flashing the smartphone with CyanogenMod version 10.2 [16], an open-source operating system for smartphones and tablet 
computers based on official releases of Android that includes third-party software. We install and compile necessary applications, including cpufrequtils package that enables inspection and control of clock frequency ranges and power schemes for each processor core.

The OnePlus One smartphone is powered by a Qualcomm Snapdragon 801 (MSM89734AC) SoC that features a quad-core ARM-based Krait 400 processor running at up to $2.5 \mathrm{GHz}$ clock frequency, an Adreno 330 graphics processor, and $3 \mathrm{~GB}$ of RAM memory. OnePlus One supports a range of communication protocols including WLAN $802.11 \mathrm{a} / \mathrm{b} / \mathrm{g} / \mathrm{n} / \mathrm{ac}$, Bluetooth 4.1, USB, HDMI, and several cellular network protocols such as GSM/HSPA/LTE. OnePlus One has preinstalled CyanogenMod version 12.1 that includes third-party software modules needed for power and clock frequency management.

To prepare the smartphones for energy profiling, theirunderlying plastic shieldsareremoved or modified to reveal connections on theirmotherboards and daughter boards as shown in Figure 3. The smartphones' batteriesare removed, and power connectors fromthe battery simulator are connected instead (for OnePlus One, internal USB cable is rewired to battery connector). Connectors to smartphone components such as LCD display, touchscreen, USB, and others can be easily unplugged during power profiling, thus enabling selective profiling that excludes energy consumed by these components.

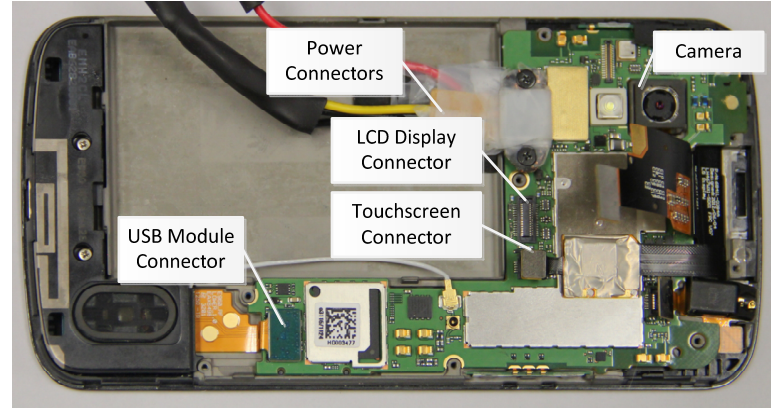

(a)

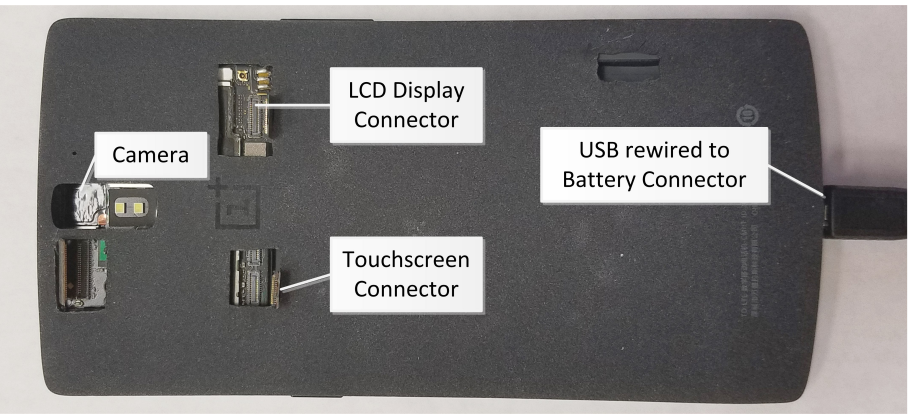

(b)

Figure 3. Nexus 4 (a) and OnePlus One (b) prepared for energy measurements

Smart Buttonis a wearable embedded platformused for automated assessment of mobility of elderly and patientssupporting automated quantifying of the 30-Second Chair Stand and the Timed Up and Go tests[13]. It incorporates an Arduino-compatible platform called Teensy 3.1, a 9-DoF inertial sensor, a Bluetooth radio, 
and a Li-Ion battery with a charger. The Teensy 3.1 platform features a MK20DX256VLH7 SoC with a 32bit ARM Cortex-M4 processor running at $72 \mathrm{MHz}, 256 \mathrm{~KB}$ of flash memory, and $64 \mathrm{~KB}$ of RAM memory. The entire package has a form factor of 50.8 x 28.6 x $15.9 \mathrm{~mm}$ and weighs $40 \mathrm{~g}$. The platform'sfirmware handles Bluetooth communication, sampling of inertial signals (3-axis accelerometer, 3-axis gyroscope, and 3-axis magnetometer), sending signals via the Bluetooth interface to a workstation or smartphone, on-board processing of signals to detect certain posture transitions (standing up, sitting down, walking, turning around), and deriving parameters that quantify the tests. After a test is completed, test parameters are sent to the workstation or smartphone that initiated test via the Bluetooth interface.

To prepare Smart Button for energy profiling, its battery connector is disconnected and replaced with a fitted power connector from the battery simulator, as shownFigure 4 . The connector from the battery simulator is made long enough to be able to perform tests that requirewalking short distances. One or more digital outputs from the Teensy platform are connected to the DAQ inputs. This way, the device's firmware can generate triggers that are precisely captured by the DAQ (e.g., when a new command is received over the Bluetooth interface).These triggers can then be used when processing current samples captured by the battery simulator to determine energy consumed in certain portions of the firmware.

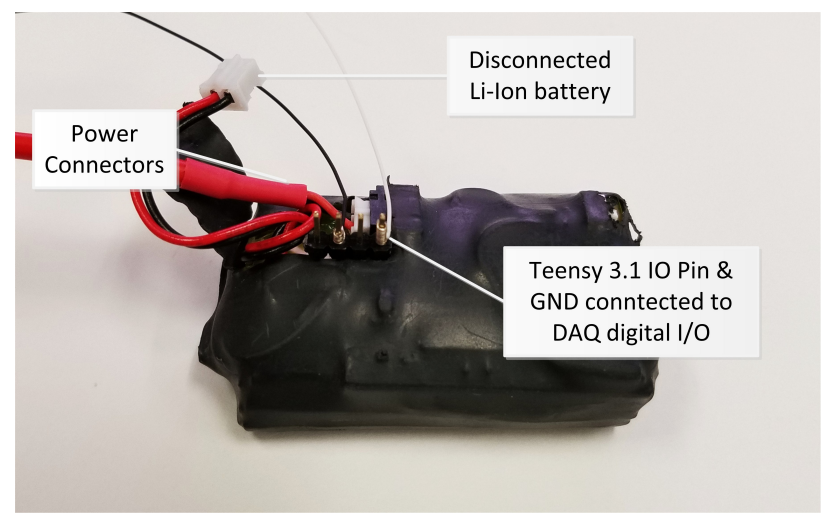

Figure 4. Smart Button prepared for energy measurements 


\section{B. Battery Simulator and DAQ}

The battery simulator and the DAQ devicereside inside an NI PXIe-1073 chassis [20], which is connected to an MXI-Express Interface card inside the workstation (Figure 2). The battery simulator is a specialized programmable power supply optimized for powering devices under test, including cellular handsets, smartphones, tablets, and other mobile devices. Its $+6 \mathrm{~V}, \pm 3$ A Channel 0 is designed to simulate a lithiumion battery cell's transient speed, output resistance, and 2-quadrant operation (source/sink) [12]. The simulator's ultrafast transient response time, $<20 \mu \mathrm{s}$, allows it to respond rapidly to changes in load current with a minimal voltage dip. It can sample the voltage and current drawn on its channels with a configurable sampling frequency of up to 200,000 samples/s and a sensitivity of the current measurements of $1 \mu \mathrm{A}$.

The DAQ device features optimized and high-performance sampling of analog, digital, and counter/timer inputs. Digital signals can be sampled at $10 \mathrm{MHz}$ rate, allowing for flexible time stamping of events generated on embedded platforms.

To power the smartphones, we configure the battery simulator's channel 0 to provide $4.1 \mathrm{~V}$ that corresponds to the voltage of the smartphone's battery when fully charged.The Nexus 4 peak current goes up to $1.5 \mathrm{~A}$, whereasthe OnePlus One current peaks at3.1 A. To power the Smart Button device, we configure the battery simulator's channel 0 to provide $3.7 \mathrm{~V}$ that corresponds to the voltage of its Li-Ion battery.

\section{Workstation}

The workstation is a Dell T7500 Precision with an Intel Xeon processor, 12 GB of system memory, running the Windows 7 Pro operating system. The workstation connects to the battery simulator and the DAQ through an MXI-Express card plugged into its PCI Express. When profiling Android mobile devices, the workstation connects to them through either a wireless LAN interface or through a wired USB interface (Figure 2). When interfacing a mobile device over a USB port, we need to take into account the energy delivered to the device through the USB port. This can be done by powering the USB from the second channel of the battery simulator. By sampling the current drawn by the device on this channel, we can 
account for the energy received through the USB. This energy is then combined with the energy measurements on channel 0 to determine the total energy. An alternative is to disconnect the USB once power profiling is launched.To simplify the profiling, in the rest of the paper we rely on the link through the WLAN interface.The workstation connects to embedded platforms indirectly through the DAQ and platform's digital outputs.

mLViewPowerProfile is our custom software tool for automated capturing of power traces and evaluating energy efficiency of mobile or embedded applications. It runs on the workstation and controls concurrently both the battery simulator (with DAQ) and the device under test. Figure 5 shows the mLViewPowerProfile's graphical user interface. A user configures the channels of the battery simulator. This involves setting the voltage and the current limits, the sampling frequency, the transient time, as well as software driver parameters that control fetching the current samples from the battery simulator. We sample the current at the maximum sampling rate of 200,000 samples/s, but we choose to average 10 samples, thus recording 20,000 samples per second in a user-specified file (appsSamples.txt). 


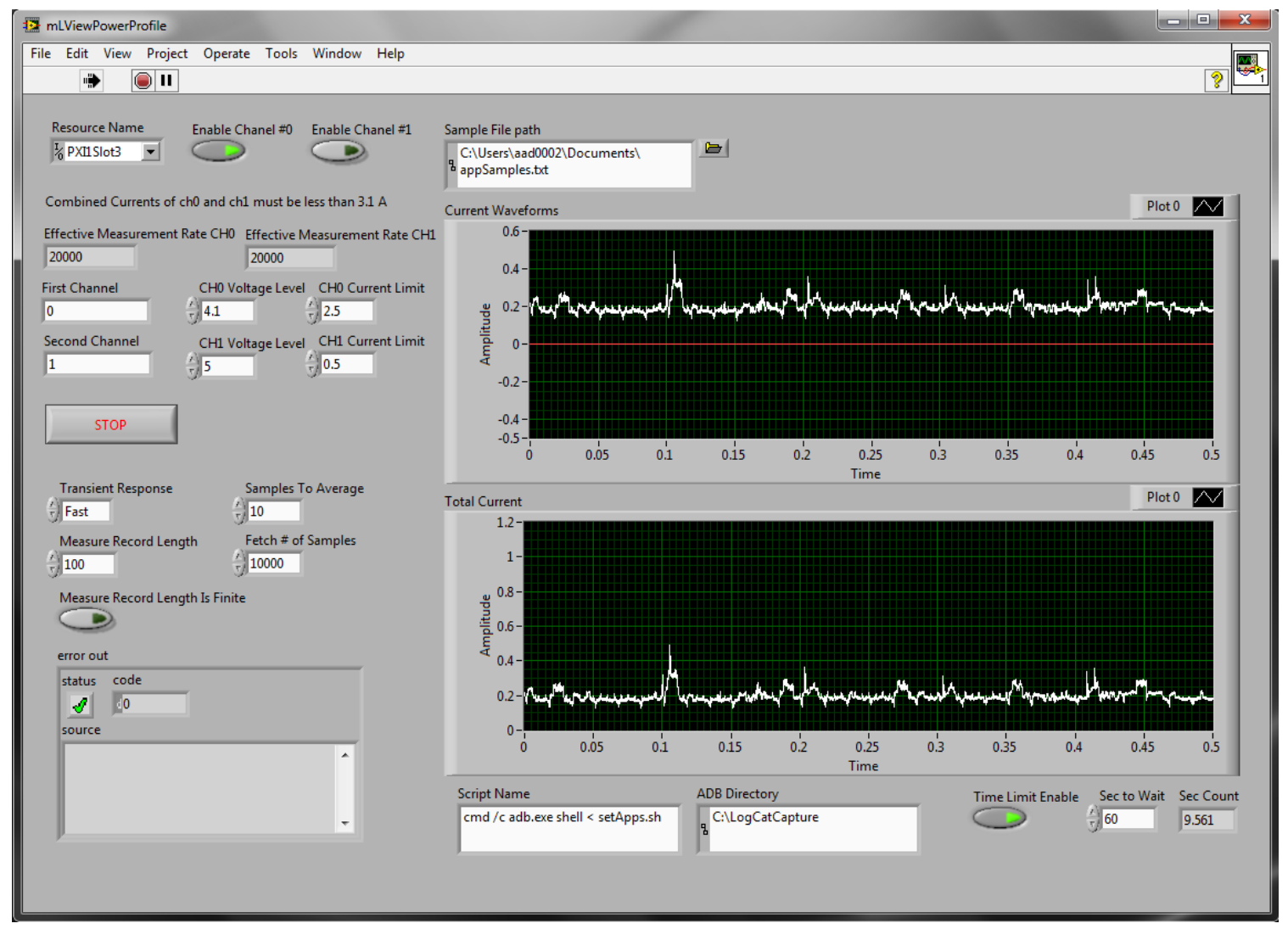

Figure 5. mLViewPowerProfile user interface

The communication with Android devices under test is carried out over the Android Debug Bridge (adb) [21]. $a d b$ is a client-server program that includes the following components: a client, which runs on the workstation; a server, which runs as a background process on the workstation; and a demon, which runs on the device. The user establishes an $a d b$ connection and runs a script command file that invokes applications or activities that need to be profiled. The following section demonstrates profiling of Android applications.

\section{Power Profiling OF ANDroid APPliCATIONS}

This section describes methods for power profiling of applications running on Android devices. SectionsIII.Aand III.B describe profiling of uninstrumented and instrumented Android applications, respectively. Section III.C describes profiling of Android application under CyanogenMod. 


\section{A. ProfilingUninstrumented Android Applications}

To detect points of interest for energy profiling, the Android logging system is used. As an example, we will consider playing a video located in the smartphone's file system.

The first step is to launch a script file to be executed on the smartphone. mLViewPowerProfile starts a Windows command shell (cmd) that invokes the $a d b$ shell ( $a d b)$ and the script file (setupApps.sh) as shown in Figure 5 (see Script Name command box). Figure 6 shows the content of the setApps.sh script file. The first command sets the smartphone's working directory where the video file is located (/sdcard/test). The second command invokes a command file for playing video, runPlayVideo.sh. This script file is prepared in advance and placed in the working directory on the smartphone. The run video script is executed with the nohup command, thus ensuring that its execution continues even when we exit the adb shell. The last two commands are used to exit the $a d b$ shell and the Windows command shell.

1. cd /sdcard/test/

2. nohup ./runPlayVideo.sh \&

3. exit

4. exit

Figure 6. ADB Shell script (setApps.sh)

The script file for playing a video file is shown in Figure 7. The first line sets a $5 \mathrm{~s}$ delay during which the smartphone is in the idle state, and the current drawn by the smartphone corresponds to the idle current ( $\left.\mathrm{I}_{\mathrm{IDLE}}\right)$. The command in line 2 uses the Android's activity manager, am, to start an activity that plays a video file called FlyingBirds.mkv. The video is played for approximately $24 \mathrm{~s}$, followed by another delay that puts the smartphone in the idle state for $5 \mathrm{~s}$ after the activity is completed.

1. sleep 5

2. am start - $n$ com.android.gallery3d/.app.MovieActivity - $d$

'file:///sdcard/test/FlyingBirds.mkv'

3. sleep 24

4. sleep 5

Figure 7. Run-script for playing a video FlyingBirds.mkv 
mLViewPowerProfile captures a global timestamp that corresponds to the first sample and logs the current samples in a sample file on the workstation during the execution of the entire script $(\sim 35 \mathrm{~s})$. To determine the current samples that correspond to the beginning and the end of the activity profiled, the smartphone and the workstation are synchronized using the network synchronization protocol and the Android logging system is used to record global timestamps of events of interest for the profiled application.

The Android logging system provides a mechanism for collecting and viewing system debug output [22]. Logs from various applications and portions of the system are collected in a series of circular buffers, which then can be viewed and processed by the logcat command in the adb shell. Typically, the circular buffers are cleared before the profiling is conducted using logcat $-c$ command. After the test is completed, we use logcat to extract the log messages including timestamps using the following command: logcat $-d-v$ time > logcat_output.txt.

Figure 8 shows an excerpt ofthe logcat output from Nexus 4 with messages that are relevant to the profiling task. The timestamp of the beginning of the script execution is 18:55:49.633, the activity manager is started approximately $5 \mathrm{~s}$ later at 18:55:54.873, the video starts playing at 18:55.55.624 and ends at 18:56:19.457. Thus, the video playing activity takes 23.8 s. Similar logcat output is extracted from the profiling task on the OnePlus One, with the video playback taking $24.5 \mathrm{~s}$.

Figure 9 and Figure 10show the measured current drawn by the Nexus 4 and OnePlus One smartphones, respectively,during the execution of the runPlayVideo.sh script file. The Start am, Start video, and Finish video markers illustrate the timestamps that correspond to the moments when the am command is issued, the video starts playing, and when the video finishes playing, respectively. The graphs on the bottom show the filtered waveforms, provided here only to enable easier visual inspection of the changes in the current drawn. The unfiltered samples, shown in Figure 9, topand Figure 10, top are used to calculate the total energies and the energy overheads. 
1. - - - - - beginning of /dev/log/main

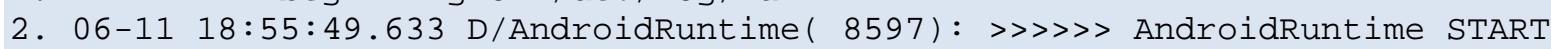
com.android.internal.os. RuntimeInit $\ll<<<$

3. .......

4. --..-- beginning of /dev/log/system

5. $06-11$ 18:55:54.873 I/ActivityManager( 644): START u९ \{dat=file:///sdcard/FlyingBirds.mkv flg=0x1000000๑ cmp=com.android.gallery3d/.app.MovieActivity $\}$ from pid 8631

6. . . . .

7. 06-11 18:55:55.624 E/OMX-VDEC-1080P( 194): In OMX vdec Constructor

8. . . .

9. 06-11 18:56:19.457 E/OMX-VDEC-1080P( 194): Exit OMX vdec Destructor

Figure 8. Log messages captured on the smartphone during power profiling of video playing activity.

To find the total energy consumed while playing the video, we first determine offsets of the recorded timestamps relative to the timestamp that is captured by mLViewPowerProfile at the beginning of the measurement $\left(\mathrm{T}_{\mathrm{INIT}}=0 \mathrm{~s}\right)$. Next, we determine indices of the current samples that correspond to the beginning (N.SS) and the end (N.ES) of the execution, calculated from the starting and ending timestamps and the sampling frequency, $F_{S}$, as shown in (1) and (2). The total energy, ET, is then calculated as shown in (3).

$$
\begin{gathered}
N . S S=T_{\text {START }} \cdot F S \\
N . E S=T_{E N D} \cdot F S \\
E T=\sum_{j=N . S S}^{N . E S} I_{j} \cdot V_{\text {SUPPLY }} \cdot \Delta t, \Delta t=\frac{1}{F_{S}}
\end{gathered}
$$

Nexus 4: Playing video from sdcard (Raw)

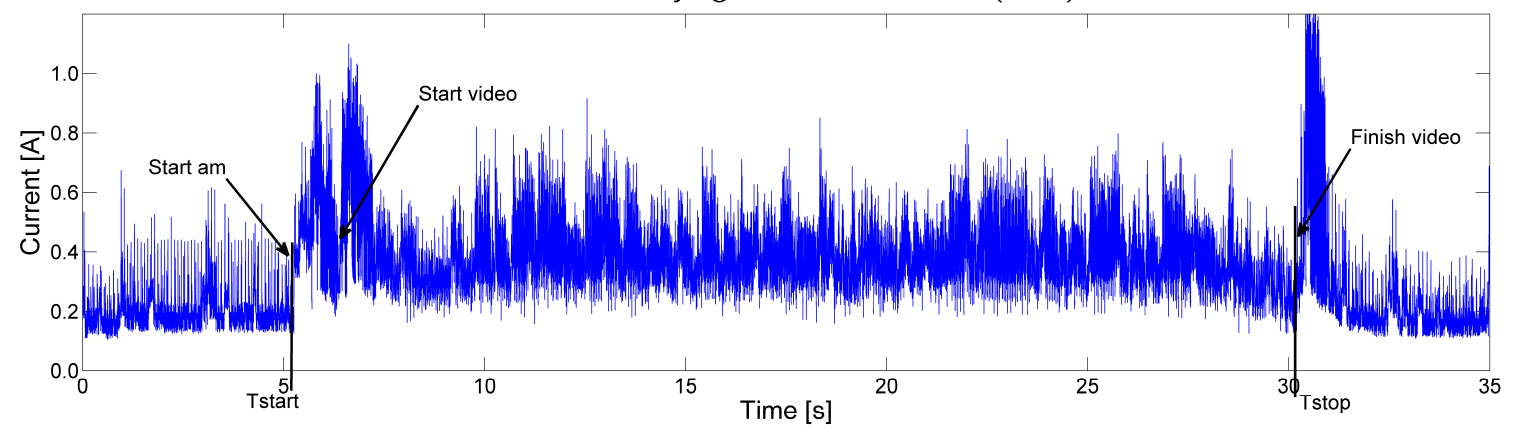


Nexus 4: Playing video from sdcard (Filtered)

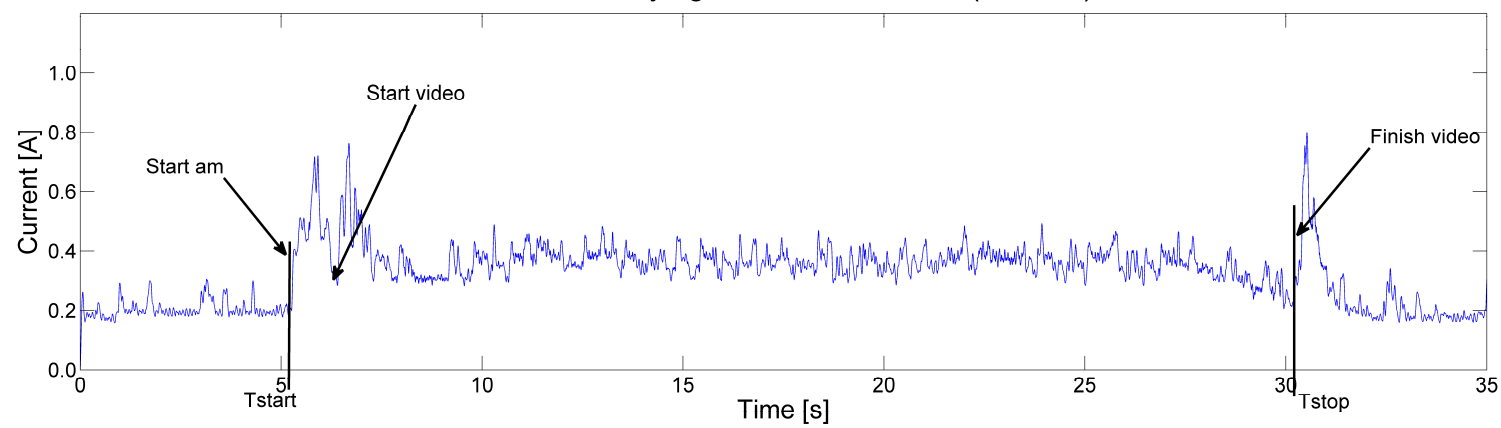

Figure 9. Current drawn by Nexus 4while playing video

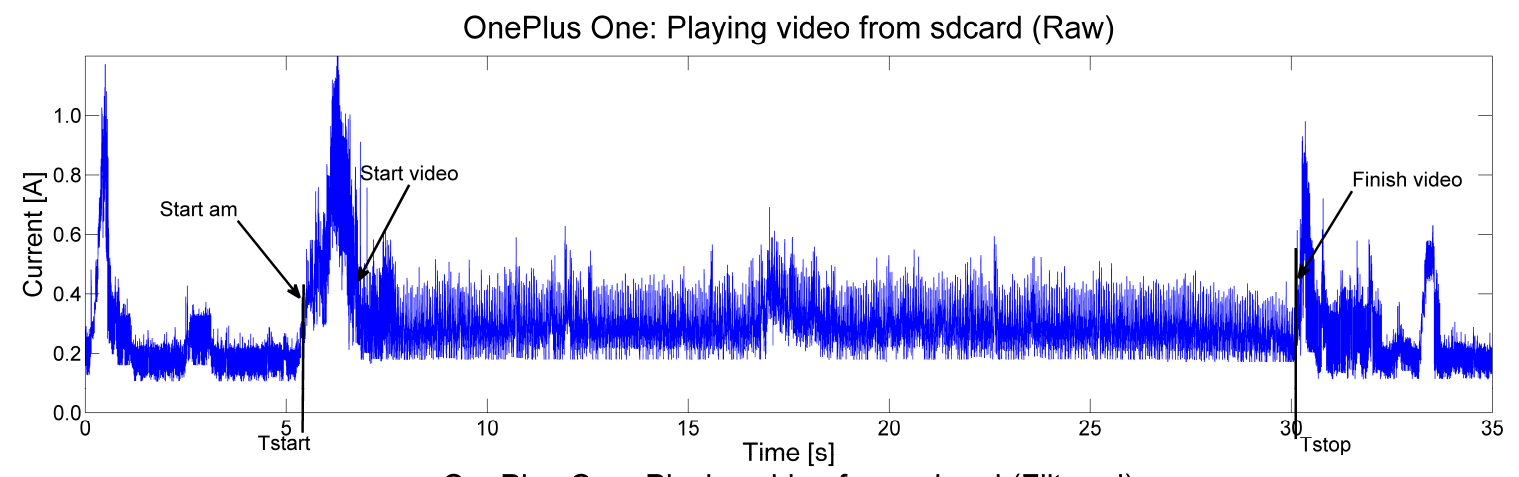

OnePlus One: Playing video from sdcard (Filtered)

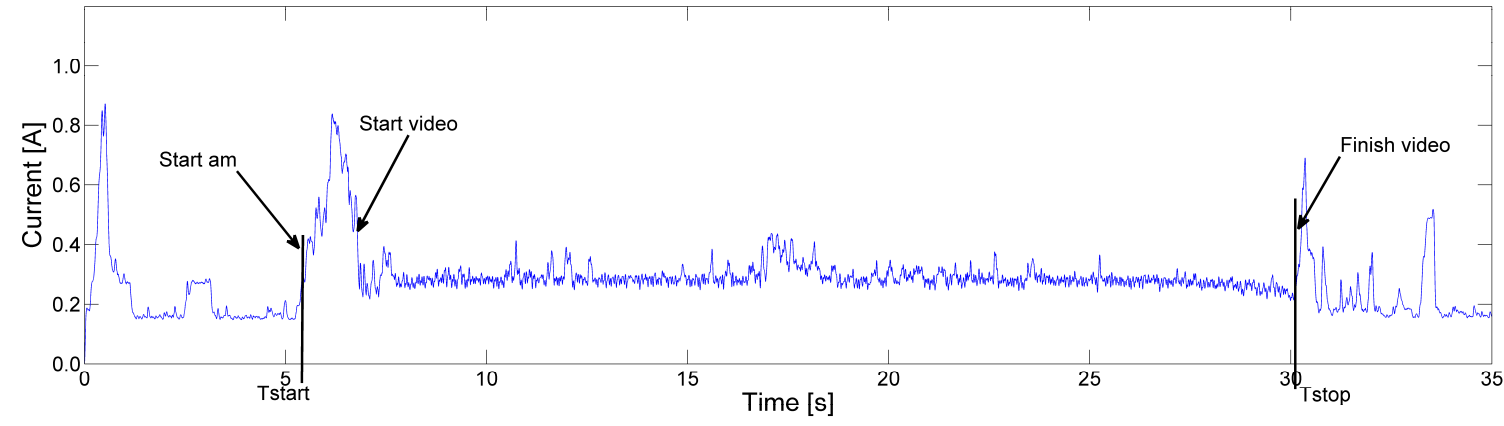

Figure 10. Current drawn by OnePlus Onewhile playing video

In addition to the total energy we can calculate the energy overhead, EO, caused by the executing program alone, which excludes the energy spent when the smartphone is in the idle state. The energy overhead is calculated as shown in (4).

$$
E O=E T-I_{I D L E} \cdot V_{S U P P L Y} \cdot\left(T_{E N D}-T_{S T A R T}\right)
$$

A PERL script takes the processed timestamps and the file with the current samples as inputs and calculates the energies. The total energy for playing video on Nexus 4 (from Start am to Finish video) is 
36.01 $\mathrm{J}$, and the energy overhead is $16.72 \mathrm{~J}$. If we measure the energy from the moment the video starts playing (from Start video to Finish video), the energies are 34.91 J and 15.38 J. The total energy for playing video on OnePlus One is $30.83 \mathrm{~J}$, and the energy overhead is $14.92 \mathrm{~J}$. Measuring from the video start, the energies are $27.21 \mathrm{~J}$ and $12.48 \mathrm{~J}$. In both cases, the measurementsare conducted with active LCD displays at the same brightness level and the WLAN interfaces enabled.

To determine the impact of powering the LCD displays alone, the experiment is repeated with the LCD displays disconnected. The total energy for playing the video on Nexus 4 is $26.49 \mathrm{~J}$ and the overhead is 15.17 J. The total energy on OnePlus One is $21.71 \mathrm{~J}$ and the overhead is $13.62 \mathrm{~J}$.These results show that the display alone takes a significant amount of the total energy when active on both smartphones. However, a small difference in the energy overheads (16.72 vs. $15.17 \mathrm{~J}$ and 14.92 vs. $13.62 \mathrm{~J})$ indicates that playing the video does not increase significantly the energy consumed by the displays relative to its usual consumption when active.The results also show that OnePlus One consumes less energy during video playbackwhich can be attributed to its more energy efficient LTPS LCD display.

\section{B. Profiling Instrumented Android Applications}

In this section, we look at power profiling using our environment from a developer's perspective. Here we assume that a developer wants to determine energy-efficiency of a certain activity or its segment. Instead of relying on Android system log messages, the developer instruments the source code so that timestamps are generated and logged at points of interest in the application lifetime.

To help guide energy profiling of particular segments of applications, software developers can instrument their Android applications by inserting custom log messages. Android log messages are divided into several categories. For example, Log.e() is used for logging serious errors, Log.w() for reporting system warnings, Log.i() for information logging (e.g., successful connection), Log.d() for debugging messages, and Log.v() for all other verbose messages (e.g., entering a function). Each message can be marked by a custom tag. 
A typical Android application consists of different activities that load GUI elements, start various functions, services, threads, asynchronous tasks, and intents and provide user interaction via buttons and other GUI elements that lead to a transition from one activity to the next. Using custom log messages a developer can instrument any part of the application. Particularly, developers may utilize the Android lifetime cycle's state methods such as onCreate(), onStart(), onResume(), onStop(), onDestroy(), and onRestart(). For example, onCreate() is called at the initial start of the activity, while onDestroy() is called at the end of the activity.

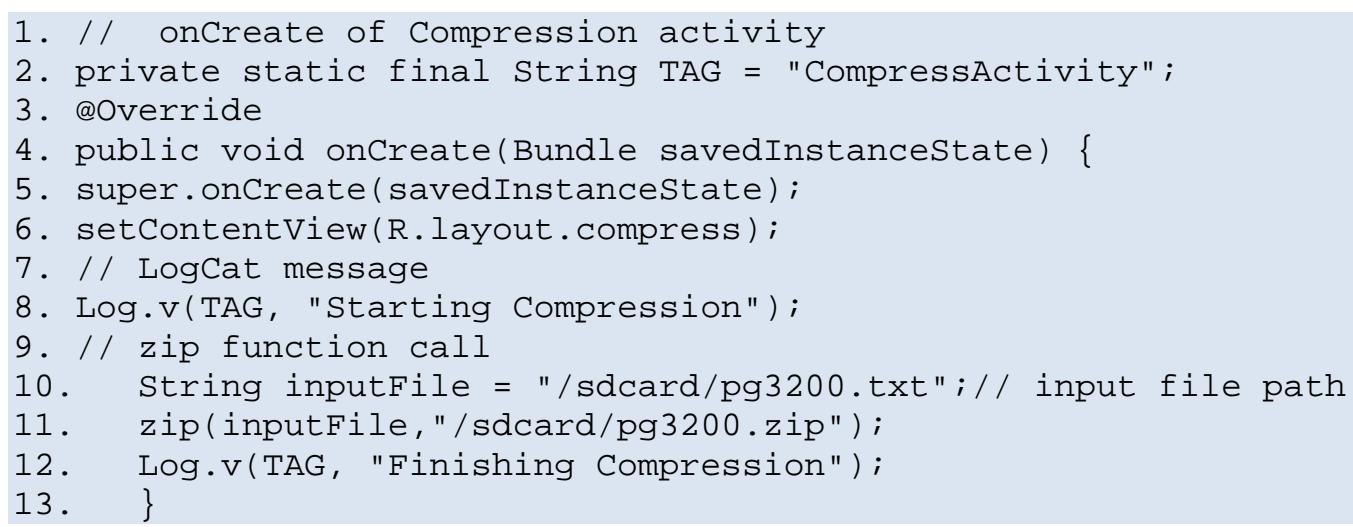

Figure 11. Instrumenting onCreate() method with verbose log messages

To illustrate this approach, we develop a test Android application, called testZip. testZip compresses an input file using Android's ZipOutputStream class. Figure 11 shows its onCreate() method which calls the zip method right after opening and loading application layout on the screen. The zip function compresses an input file and writes the compressed file in the internal file system. To capture execution time of this function, the Log.v() messages are inserted before the compression (line 8) and after the compression (line 12). Figure 12 shows an excerpt from the Nexus 4 logcat output with custom messages from which the starting and ending timestamps can be extracted and used in energy calculations. Similar logcat output is extracted when profiling OnePlus One.

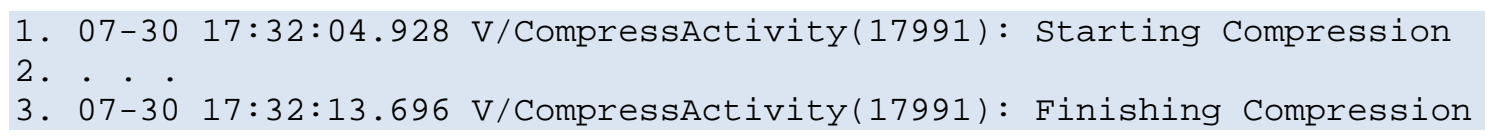

Figure 12. Log messages with a custom CompressActivity tag 

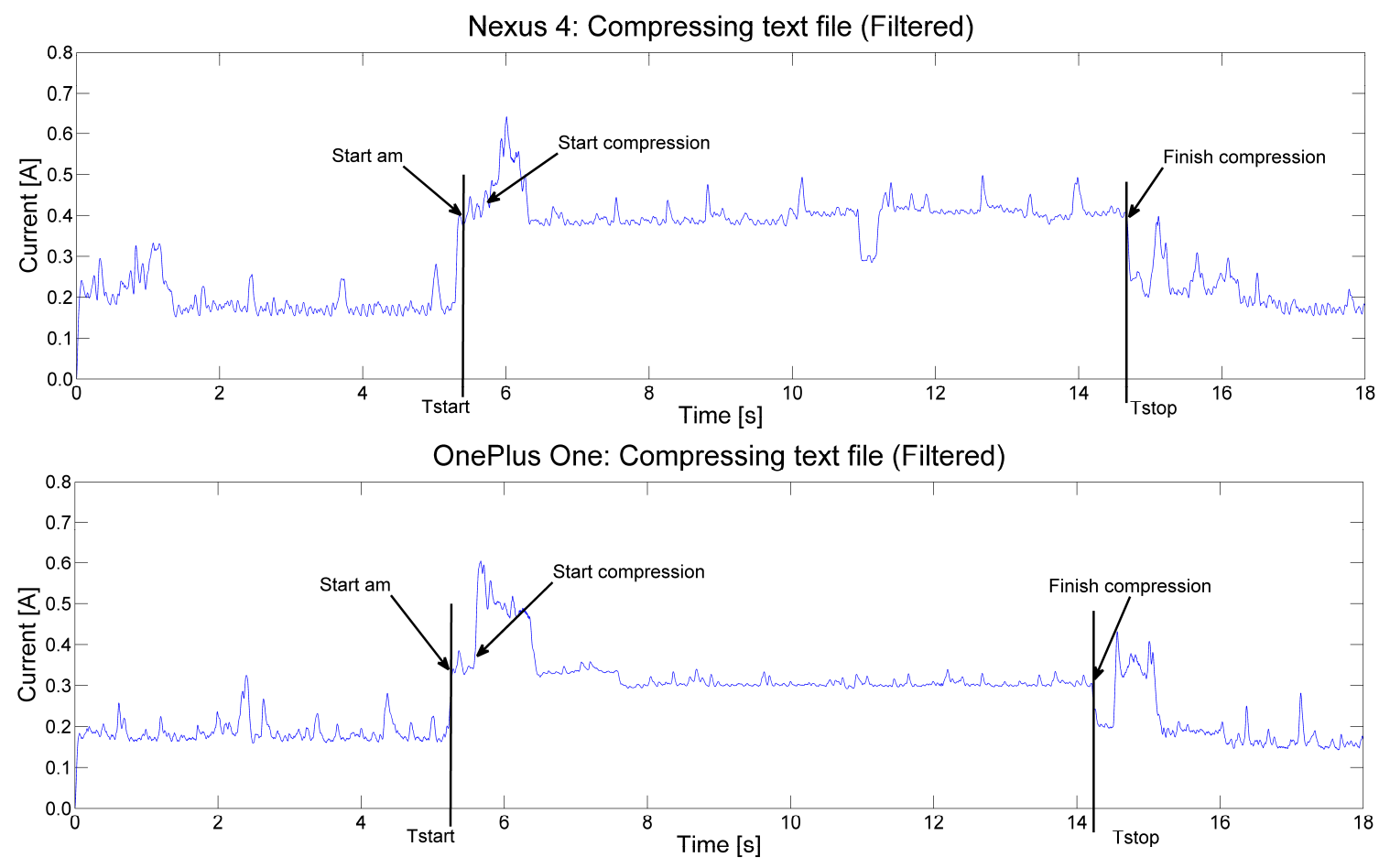

Figure 13. Current drawn by Nexus 4 (top) and OnePlus One (bottom) while compressing an input file

Figure 13 shows the filtered measured current drawn by the Nexus 4 and OnePlus One smartphonesduring the execution of a compression test testZip application. The compression is preceded and followed by 5 second delays. The Start compressionand Finish compressionmarkers illustrate the custom timestamps recorded inside the application before the very start of the compression activity and after the compression activity is completed. For Nexus 4, the activity manager starts the test application and its interface on the screen at $\mathrm{T}_{\text {START_AM }}=5.45 \mathrm{~s}$, and the compression activity itself starts at $\mathrm{T}_{\text {START_COMP }}=5.82 \mathrm{~s}$ and finishes at $\mathrm{T}_{\text {FINISH_COMP }}=14.59 \mathrm{~s}$. For OnePlus One, the activity manager starts the test application and its interface on the screen at $\mathrm{T}_{\mathrm{START}_{-} \mathrm{AM}}=5.24 \mathrm{~s}$, and the compression activity itself starts at $\mathrm{T}_{\mathrm{START}}$ COMP $=5.85 \mathrm{~s}$ and finishes at $\mathrm{T}_{\text {FINISH_COMP }}=14.23 \mathrm{~s}$. The total energy for compressing the input file on Nexus 4 is $15.28 \mathrm{~J}$ (14.62 $\mathrm{J}$ for compression itself), whereas the overhead energy is $8.17 \mathrm{~J}(7.80 \mathrm{~J})$. The total energy onOnePlus One is $12.09 \mathrm{~J}(11.61 \mathrm{~J}$ for compression itself) and overhead energy is $5.45 \mathrm{~J}(5.22 \mathrm{~J})$. In both cases, the measurementsare conducted with active LCD displays at the same brightness level and the WLAN interfaces enabled. 


\section{CyanogenMod Android}

In this subsection, we look at power profiling of applications from CyanogenMod Android. The main difference is that instead of using the Android logging system for extracting timestamps of relevant events, we use \$EPOCHTIME bash variable to capture timestamps and write them into a file.

Figure 14 shows a run script, runDownloadFile.sh, which downloads a text file from a server using the wget utility. The text file of 15,711,660 bytes (pg3200.txt) contains the Project Gutenberg Works of Mark Twain. The file download is preceded and trailed with 5 second delays (lines 2 and 6 in the script file) that put the smartphone in the idle state. Lines 1, 3, 5, and 7 invoke \$EPOCHTIME bash variable to generate timestamps with nanoseconds resolution that mark the entering of the script, the moment just before the file download is started, the moment when the file has finished downloading, and the moment when the script is finished. The timestamps are logged in a text file (timestamps.txt) and used in energy calculations as described above.

1. cat \$EPOCHTIME>>/data/test/timestamps.txt;

2. sleep 5

3. cat \$EPOCHTIME>>/data/test/timestamps.txt;

4. wget -qP/sdcard/ http://lacasa.uah.edu/portal/tmp/pg3200.txt

5. cat \$EPOCHTIME>>/data/test/timestamps.txt;

6. sleep 5

7. cat \$EPOCHTIME >>/data/test/timestamps.txt;

Figure 14. Run-script for downloading text over WLAN

Figure 15, top, and Figure 16, top, show the filtered measured currents during the execution of the runDownloadFile.sh script file on the Nexus 4 and OnePlus One smartphones. The smartphones connect to the local router (Linksys E900 Wireless N-300) over the WLAN interface. The Start and Finish markers illustrate the timestamps recorded in the experiment just before the start of the file download (line 3 in the script file) and right after the file downloading has finished (line 5 in the script file). The current drawn, IIDLE, when the smartphonesare idle with the LCD displays off are 0.10 A for Nexus 4 and 0.07 A for OnePlus One. The total energy consumed to download the file on Nexus 4 is $4.85 \mathrm{~J}$ and the energy overhead is $3.36 \mathrm{~J}$. The 
total energy and energy overhead on OnePlus One are $2.49 \mathrm{~J}$ and $1.73 \mathrm{~J}$, respectively. From thosemeasurements, we can use the total energy to calculate energy efficiencies (EE) for Nexus 4 and OnePlus Onedefined as the number of Megabytes transferred per Joule of energy consumed, EE(raw download $)=3.09 \mathrm{MB} / \mathrm{J}$ for Nexus 4, and $\mathrm{EE}(\mathrm{raw}$ download $)=6.02 \mathrm{MB} / \mathrm{J}$ for OnePlus One[23].The measurements indicate that OnePlus One is more energy efficient than Nexus 4.
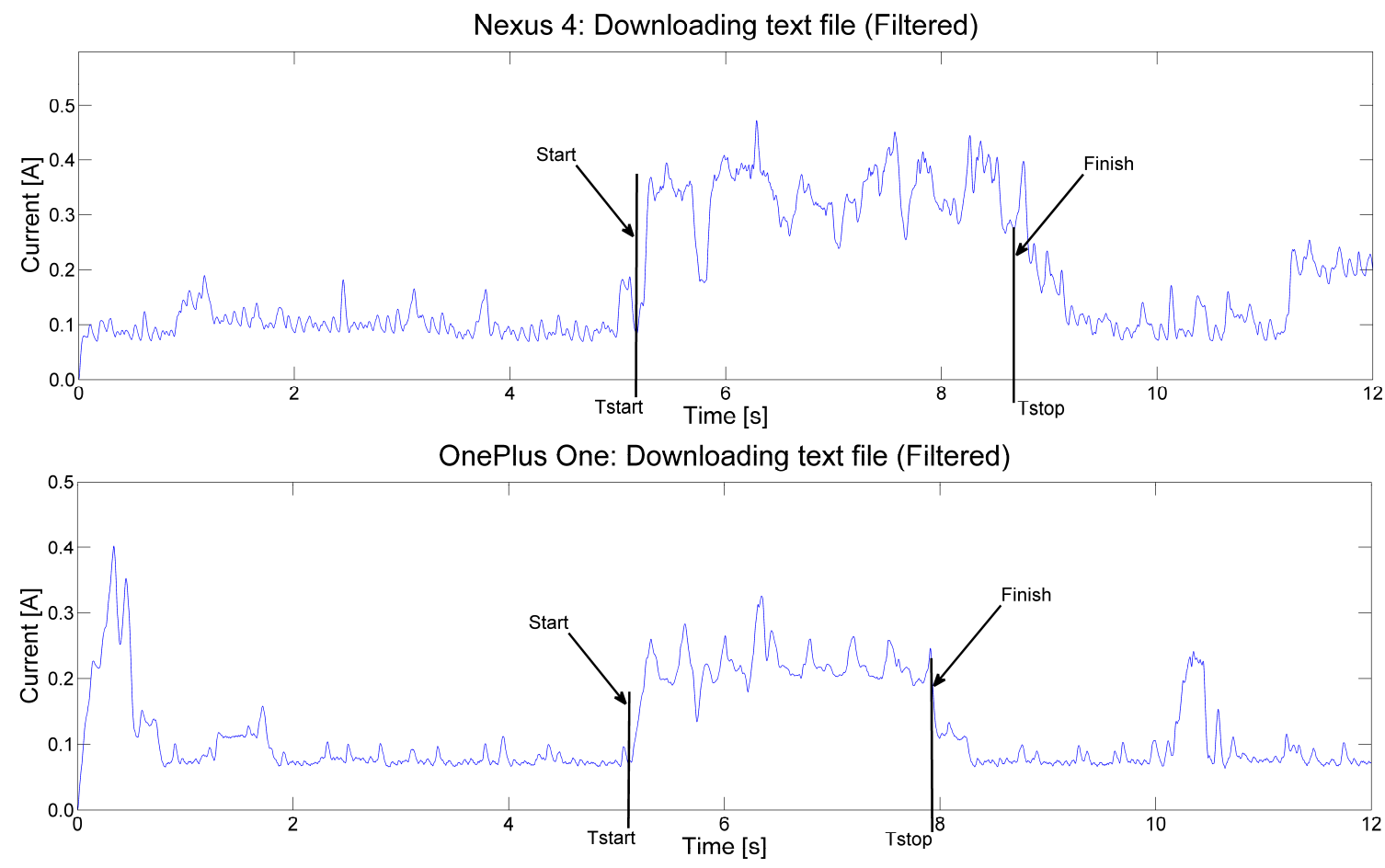

Figure 15. Current drawn by Nexus 4 (top) and OnePlus One (bottom) when downloading a raw text file

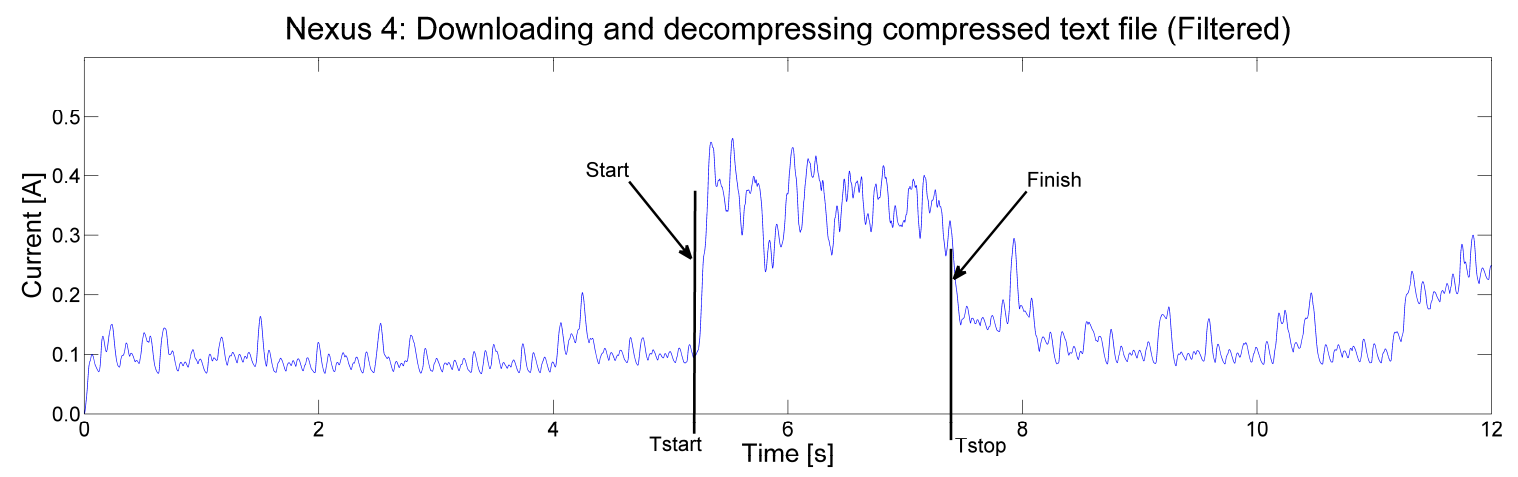




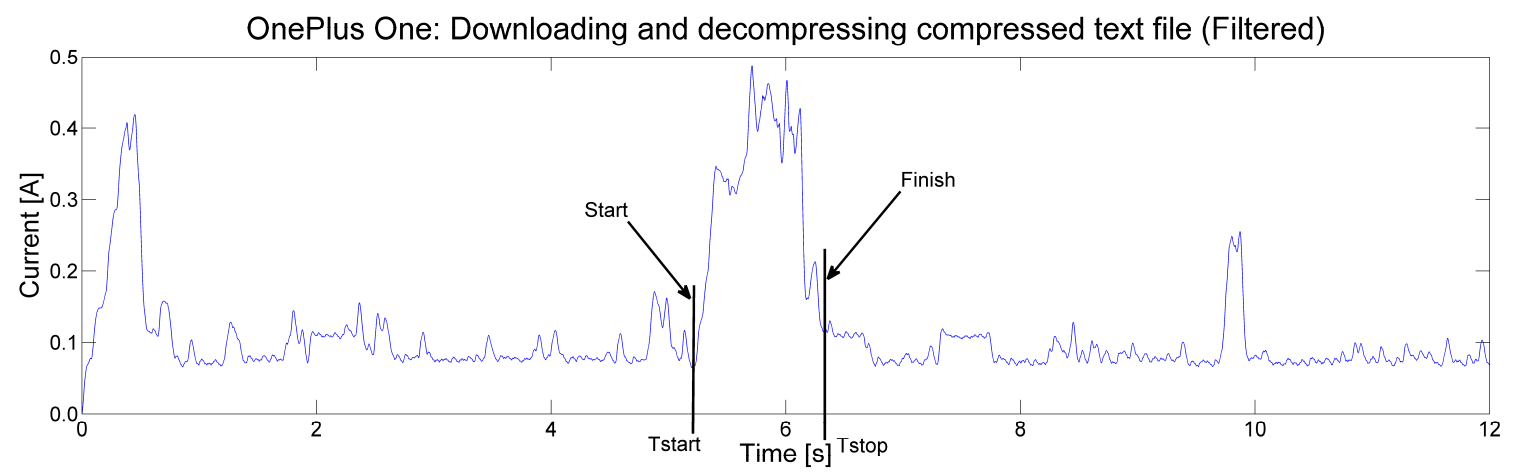

Figure 16. Current drawn by Nexus 4 (top) and OnePlus One (bottom) when downloading a compressed file with decompression (using gzip utility)

\section{POWER PROFILING OF EMBEDDED FIRMWARE}

In this section, we look at power profiling of a firmware running on an embedded system. Instead of usingAndroid built-in functions to aid synchronization of applications running on embedded platforms and power profiling tasks, synchronization is carried out by instrumenting embedded applications at points of interest. The instrumentation relies on inserting commands that write or read digital $\mathrm{I} / \mathrm{O}$ ports of the embedded platform. We assume that these ports arededicated to controlling power profiling tasks.

As one example of power profiling we consider Smart Button when executing an application that quantifies 30-Second Chair Stand test. The standard test outcome is the number of stand-ups a person can take in a 30 second period. Our application also extracts a number of other parameters including duration of each stand-up and sit-down posture transitions as well as angular velocities during these transitions. The platform is mounted on the subject's chest before the test. The application running on the smart buttonwaits to receive a Bluetooth message that indicates the beginning of the test. When the starting command is received, the application starts sampling signals from the inertial sensors. One version of application streams samples to a workstation over the Bluetooth link where further processing takes place. An alternative version performs on-platform processing of inertial signals to extract parameters of interest. The on-platform signal processing looks for characteristic patters in inertial signals that correspond to posture transitions. The end of the test occurs when a sit-down posture transition is detected. At the end of the tests, the extracted parameters 
are sent back over Bluetooth link to the smartphone or the workstation that is used to control Smart Button. To determine the energy consumed by the application, we need to timestamp important events - when the Start command is received and when the end of the test is detected. These events should be synchronized with current samples captured by the battery simulator to enable accurate power profiling.

One approach to carry out this synchronization is to instrument application to assert a digital output on the platform when the Start message is received and when the end of the test is detected. This digital output is connected to the DAQ device that samples the input.mLViewPowerProfile file stores both the current samples from the battery simulator and samples from the DAQ. This way we can identify the starting and ending current samples that correspond to the profiled code segments of interest.

Figure 17 shows the current drawn by Smart Button for the following scenarios. The top graph shows the current drawn when the platform is idling waiting for the start command over its Bluetooth interface. The graph in the middle shows the current drawn when the start command is received and raw data samples from inertial sensors are streamed to the workstation. Finally, the bottom graph shows the current drawn when the 30SCS application performs on-platform signal processing and parameter extraction. The graph markers illustrate time stamps acquired through the DAQ.

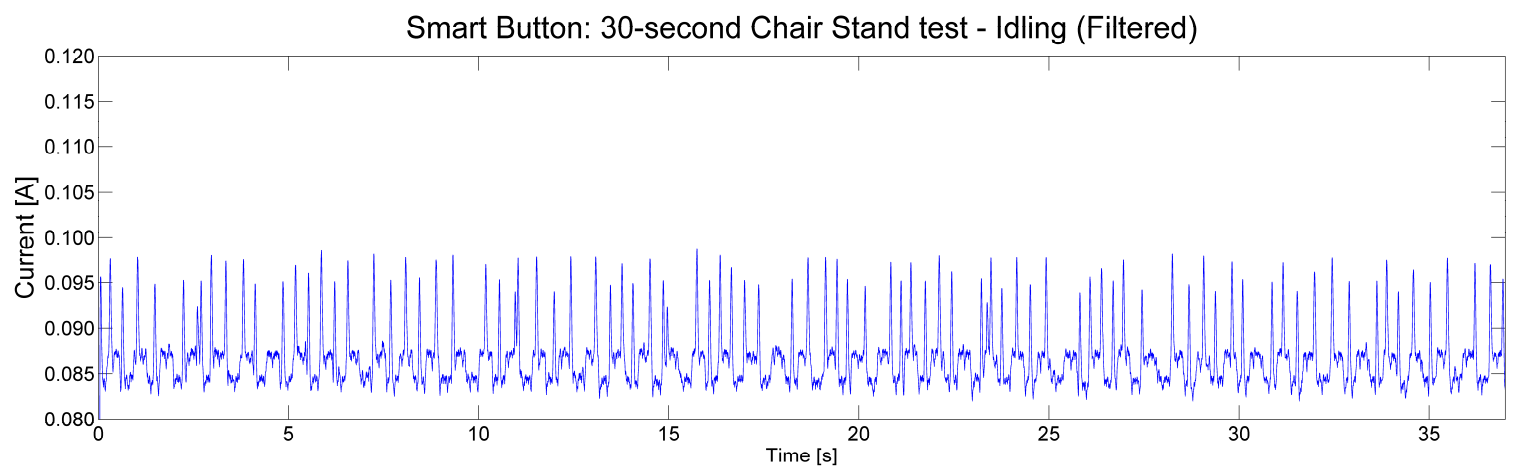



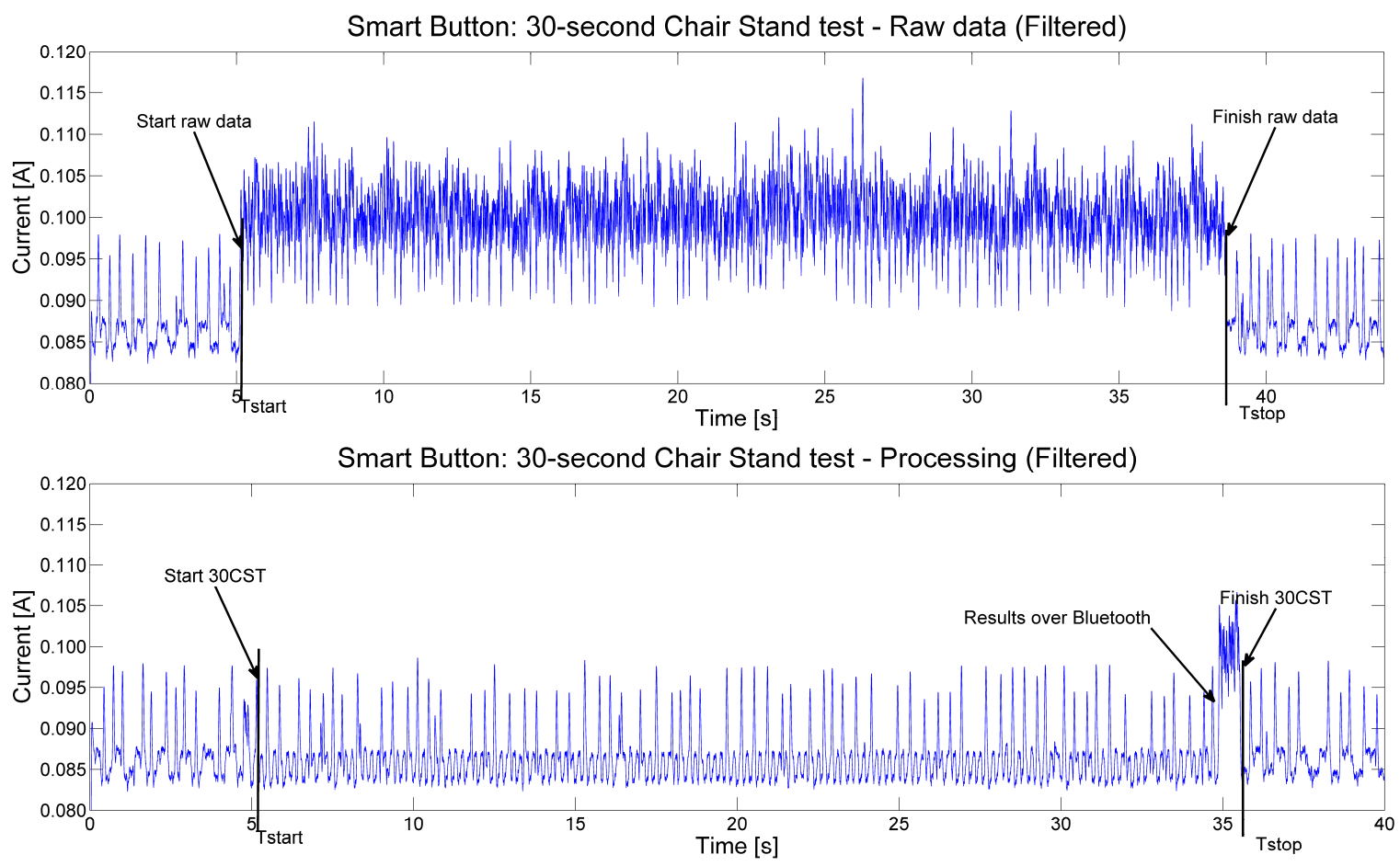

Figure 17. Current drawn by Smart Button for 30SCS application while waiting for a start command (top), streaming 9DOF inertial sensor data over the Bluetooth (middle), and executing30-Second Chair Stand test with on-board signal processing.

\section{CASE Studies}

This section demonstrates how the measuring setup can be used to quantify energy needs and improve energy-efficiency of Android applications.

\section{A. To Compress or Not to Compress}

Global mobile data traffic has grown exponentially in the last several years and reached 3.7Exabyte per month in 2015, which is over 44times greater than the total Internet traffic in 2000 [24]. Data compression is crucial in mobile data communication. It can help improve operating time, lower communication latencies, and make more effective use of available bandwidth and storage. Whereas media data such as video or audio can tolerate lossy compression that typically achieves high compression ratios, other types of data typically consumed on mobile devices such as binaries, medical data, emails, e-books rely on lossless compression that achieves modest compression ratios. 
Whether data compression reduces latency and energy consumption or not on a particular mobile device depends on many factors. Those factors include a type of communication interface (e.g., Bluetooth, WLAN, cellular), communication bandwidth, energy costs of communication, the level of redundancy in the data, and computational complexity and energy costs of a given compression or decompression utility.

In this case study we shed more light on this problem by comparing the energy and performance costs associated with downloading an e-book from the Internet. We have already determined the energy costs of downloading the uncompressed file with the Project Gutenberg Works of Mark Twain. Using our environment we measure the time and energy consumed when the compressed file is downloaded using wget and piped into the gzip decompressor that writes the uncompressed file to the file system.

Figure 18 shows a run script that downloads a compressed text file from a server using the wget utility and pipes it to the gzip utility for decompression (line 4).

1. cat \$EPOCHTIME >>/data/test/timestamps.txt;

2. sleep 5

3. cat \$EPOCHTIME>>/data/test/timestamps.txt;

4. wget -q0 - http://lacasa.uah.edu/portal/tmp/pg3200.txt.gz | gunzip -c > /sdcard/text.txt

5. cat \$EPOCHTIME >> /data/test/timestamps.txt;

6. sleep 5

7. cat \$EPOCHTIME>>/data/test/timestamps.txt;

Figure 18. Run-script for downloading and decompressing text file over WLAN

Figure 15, bottom, shows the current drawn by Nexus 4 during the download and decompress activity. The total energy consumed by the smartphone is $3.08 \mathrm{~J}$ and the energy overhead is $2.21 \mathrm{~J}$. The energy efficiency of this transfer on Nexus 4 is $4.86 \mathrm{MB} / \mathrm{J}$, which is over $57 \%$ improvement relative to the uncompressed data download.Figure 16, bottom, shows the current drawn by OnePlus One. The total energy is $1.43 \mathrm{~J}$ and the energy overhead is $1.13 \mathrm{~J}$. The energy efficiency of this transfer on OnePlus One is $10.48 \mathrm{MB} / \mathrm{J}$, which is over $74 \%$ improvement relative to the uncompressed data download. 


\section{B. To Scale or Not to Scale Frequency}

Modern SOCs that power mobile devices support dynamic voltage and frequency scaling where the clock frequency is adjusted in real-time to either preserve energy consumed or reduce heat generated by the chip. The cpufrequtils can be used to inspect and set clock frequencies for each processor core or change the CPU governor which determines frequency scaling policy. Nexus 4 supports a range of different clock frequencies from $384 \mathrm{MHz}$ to $1512 \mathrm{MHz}$ and it uses on-demand CPU governor as the default.OnePlus Onesupports a wider range of clock frequencies, ranging from $300 \mathrm{MHz}$ to 2,467 MHz, and uses interactive CPU governor as the default.

In this case study, we want to repeat the tests from V.A, but this time instead of using the default governors that scale the frequency based on the current load, we want to set the processor clock frequency at fixed $810 \mathrm{MHz}$ for Nexus 4 and at fixed 1,267 MHz for OnePlus One.

Table 1 summarizes the time and energies for both experiments conducted on Nexus 4 . Whereas the uncompressed download requires the same amount of time ( $3.55 \mathrm{~s})$, the total energy consumed and the energy overhead are slightly lower when running at fixed $810 \mathrm{MHz}$. However, the compressed download with decompression at $810 \mathrm{MHz}$ achieves savings of $19 \%$ in the total energy and $23 \%$ in the energy overhead. Thus, running at lower fixed frequency of $810 \mathrm{MHz}$ has proved both faster and more energy efficient than when running with on-demand frequency governor, which favors higher clock frequencies.

Table 2 summarizes the time and energies for experiments conducted on OnePlus One. In contrast to Nexus 4, the uncompressed download on OnePlus One at fixed frequency of 1,267 MHz requires slightly more time than when using the interactive governor (2.93), which translates into an increase in the total energy and the energy overhead. Similarly, the total energy and the energy overhead are higher when running at the fixed 1,267 MHz clock than when using the interactive governor. The interactive frequency governor uses timers that allow utilization of intermediate clock frequencies to better adapt to computing load. 
Table 1. Time and energy for downloading a text file from a server: a comparative study (Nexus 4)

\begin{tabular}{ccccc} 
Activity & Frequency & $\begin{array}{c}\text { Execution Time } \\
{[\mathbf{s}]}\end{array}$ & $\begin{array}{c}\text { Total Energy } \\
(\mathbf{E T})[\mathbf{J}]\end{array}$ & $\begin{array}{c}\text { Energy } \\
\text { Overhead } \\
\text { (OE) [J] }\end{array}$ \\
\hline Raw download & $\begin{array}{c}\text { Ondemand @ } \\
\text { 384-1512 MHz }\end{array}$ & 3.55 & 4.85 & 3.36 \\
\hline Raw Download & $\begin{array}{c}\text { Fixed @ } \\
810 \mathrm{MHz}\end{array}$ & 3.55 & 4.78 & 3.09 \\
\hline $\begin{array}{c}\text { Zip download \& } \\
\text { unzip }\end{array}$ & $\begin{array}{c}\text { Ondemand @ } \\
\text { 384-1512 MHz }\end{array}$ & 2.13 & 3.08 & \\
\hline $\begin{array}{c}\text { Zip download \& } \\
\text { unzip }\end{array}$ & $\begin{array}{c}\text { Fixed @ } \\
810 \mathrm{MHz}\end{array}$ & 1.72 & 2.59 & 1.79
\end{tabular}

Table 2. Time and energy for downloading a text file from a server: a comparative study (OnePlus One)

\begin{tabular}{ccccc} 
Activity & Frequency & $\begin{array}{c}\text { Execution Time } \\
{[\mathbf{s}]}\end{array}$ & $\begin{array}{c}\text { Total Energy } \\
\text { (ET) [J] }\end{array}$ & $\begin{array}{c}\text { Energy } \\
\text { Overhead } \\
\text { (OE) [J] }\end{array}$ \\
\hline Raw download & $\begin{array}{c}\text { Interactive @ } \\
\text { 300-2457 MHz }\end{array}$ & 2.78 & 2.49 & 1.73 \\
\hline Raw Download & $\begin{array}{c}\text { Fixed @ } \\
1267 \mathrm{MHz}\end{array}$ & 2.93 & 3.42 & 2.62 \\
\hline $\begin{array}{c}\text { Zip download \& } \\
\text { unzip }\end{array}$ & $\begin{array}{c}\text { Interactive @ } \\
300-2457 \mathrm{MHz}\end{array}$ & 1.07 & 1.43 \\
\hline $\begin{array}{c}\text { Zip download \& } \\
\text { unzip }\end{array}$ & Fixed @ & 1.24 & 1.57 & 1.23
\end{tabular}

\section{To Skype or Not to Skype}

Our environment for energy profiling can be used to provide insights that can help inform regular smartphone users about energy efficiency of certain services. To illustrate this we consider making a phone call to a telephone number. We can do so using (a) Android phone application over the $3 \mathrm{G}$ cellular interface, (b) Skype utilizing the 3G cellular interface, or (c) Skype using the WLAN interface. How do these options compare to each other regarding the total energy use? 
To find an answer to this question, we conduct several tests as follows. First, the Android phone application is selected as the default one for making phone calls. The caller initiates the call in a script file using the activity manager (am start -a android.intent.action.CALL tel:256xxxxxxx). The callee waits for approximately $7 \mathrm{~s}$ from the first ring to answer the call and then converses for approximately $12 \mathrm{~s}$. To ensure fairness, the second test with Skype is carried out in the same way. The Skype is made the default application formaking calls, and the Skype service is activated to avoid delays due to the application start up. The callee follows the same protocol. During these two tests, the WLAN interface is turned off. In the third test, the WLAN is turned on, and the cellular interface is turned off. The experiments are conducted on Nexus 4 and OnePlus One smartphones, and their LCD displays areset at the same brightness level.

Figure 19shows the current profiles during the tests conducted on Nexus 4. The top graph shows the filtered current traces when making the phone call using the Android phone application. We can see that the delay from the start of the activity manager and until the establishment of the conversation (including $7 \mathrm{~s}$ wait time while the callee's phone is ringing) is $\sim 15.4 \mathrm{~s}$, and the conversation is $\sim 12 \mathrm{~s}$. The total energy for completing the call is $33.06 \mathrm{~J}$, and the energy overhead is $11.66 \mathrm{~J}$. The middle graph shows the filtered current traces when making the Skype call that uses the cellular interface. We can observe a significant delay from the moment the call is launched until the moment the callee's phone start ringing of almost $35 \mathrm{~s}$. The total energy for the entire activity is $88.80 \mathrm{~J}$, and the overhead is $51.60 \mathrm{~J}$. Finally, the bottom graph shows the current traces when making the Skype call that uses the WLAN interface. In this case, the energies are slightly higher than in the case of the Android phone application.

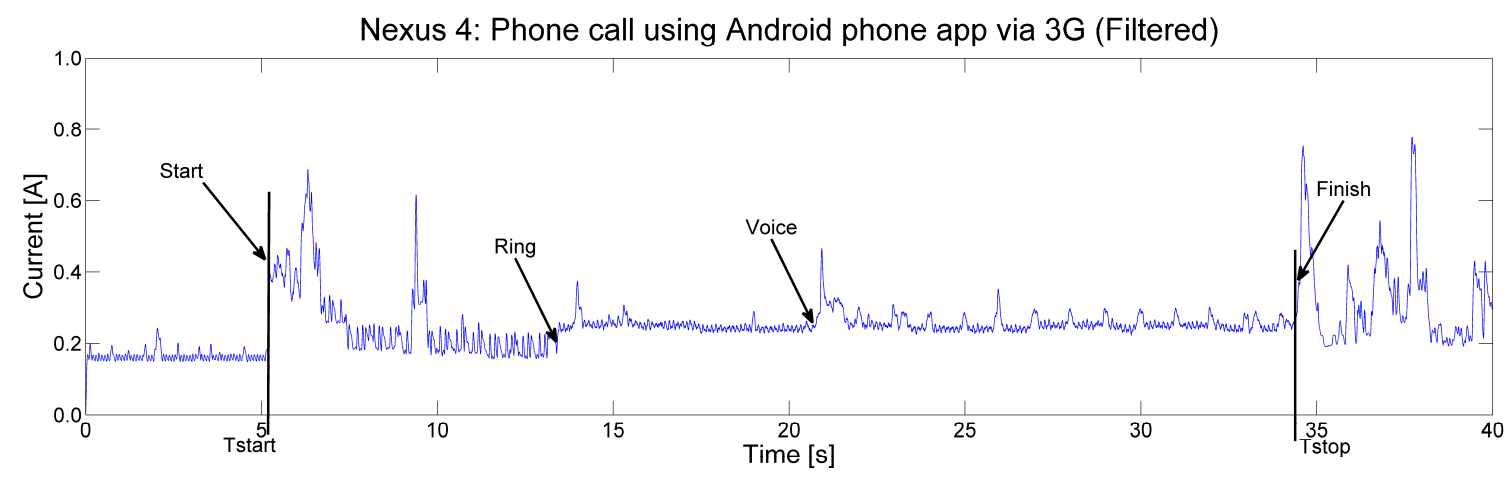



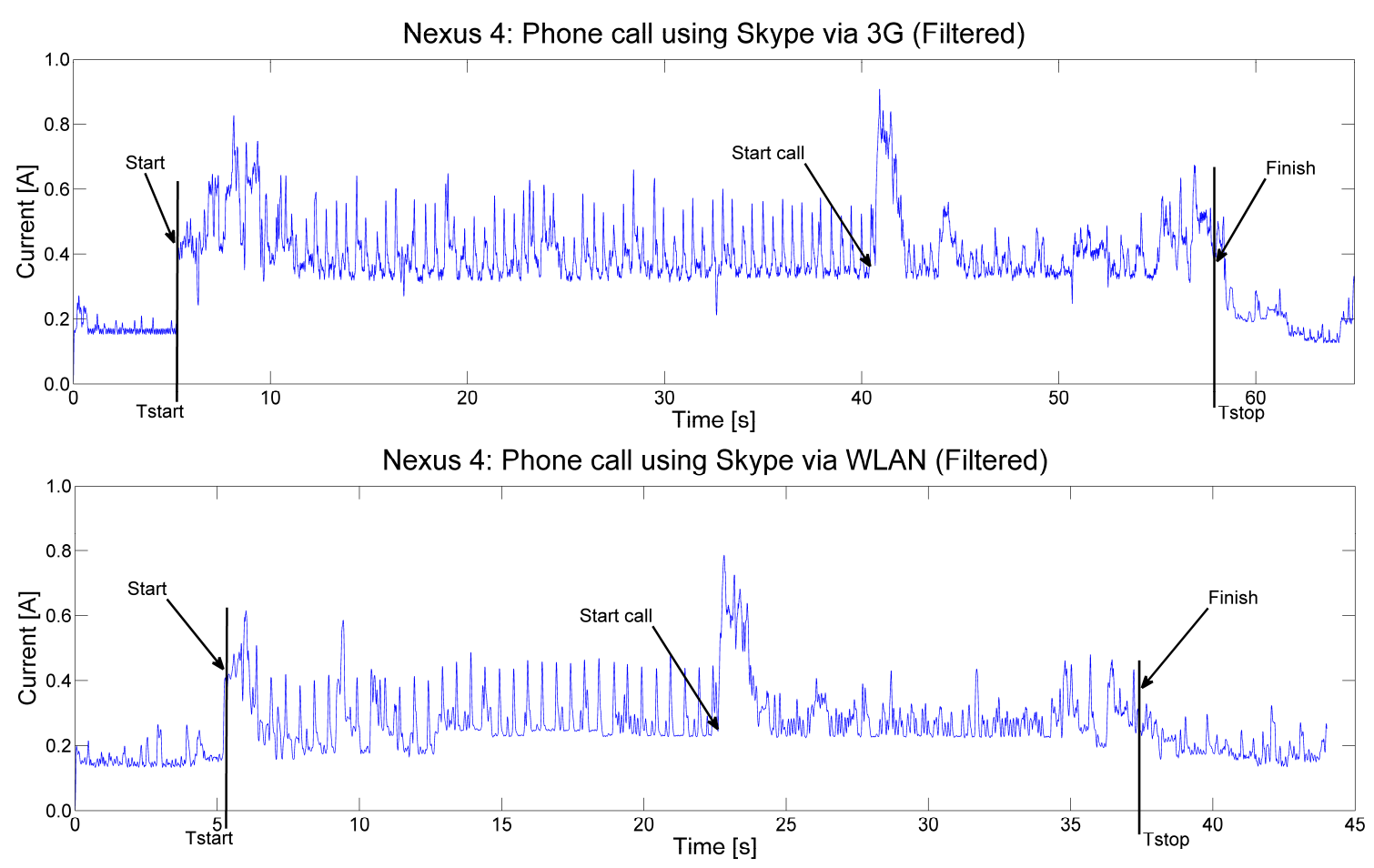

Figure 19. Current drawn by Nexus 4 while making a phone call using

Android phone application (top), Skype with cellular interface (middle), and Skype with WLAN interface (bottom)

Figure 20shows the current profiles during thetests conducted on OnePlus One. The top graph shows the filtered current traces when making the phone call using the Android phone application. The delay from the start of the activity manager to the moment when the conversation is established (including $7 \mathrm{~s}$ wait time while the callee's phone is ringing) is $\sim 15.13 \mathrm{~s}$ and the conversation is $\sim 12 \mathrm{~s}$. The total energy for completing the call is $60.97 \mathrm{~J}$ and the energy overhead is $38.78 \mathrm{~J}$. Thus, OnePlus One requires significantly more energy for making the call using the Android phone application. When using Skype over the 3G cellular interface, the delay from the moment the call is launched until the moment the callee's phone start ringing is $17.4 \mathrm{~s}$, which is significantly lower than for Nexus 4 . The total energy for the entire activity is $57.45 \mathrm{~J}$ and the energy overhead is $34.18 \mathrm{~J}$, which is less than for Nexus 4. Finally, the bottom graph shows the current traces when making the Skype call over the WLAN interface. The energies are lower than in the case of the Android phone application or the Skype over the cellular interface. 

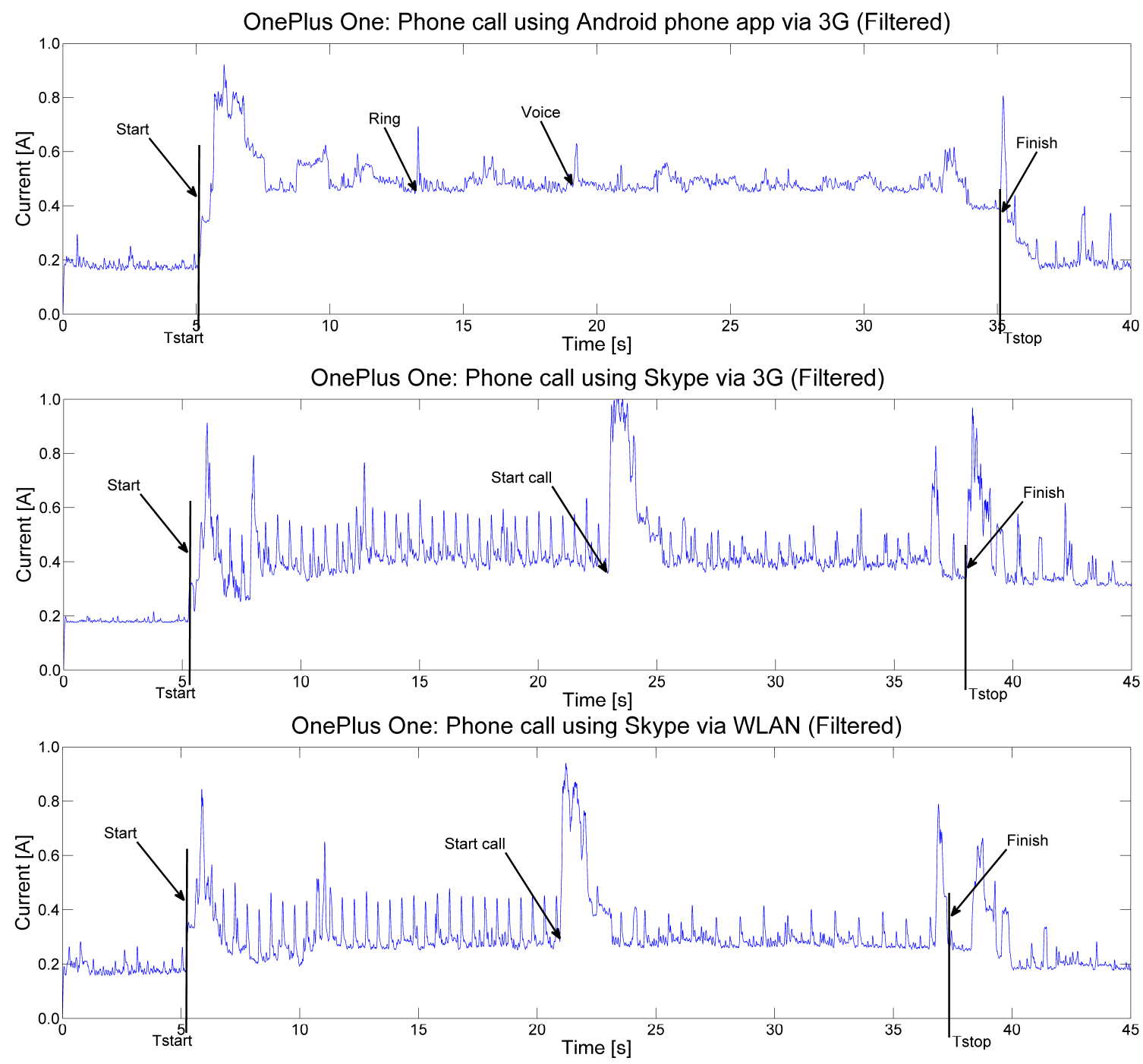

Figure 20. Current drawn by OnePlus One while making a phone call using

Android phone application (top), Skype with cellular interface (middle), and Skype with WLAN interface (bottom)

Table 3 summarizes times to establish the connections and energies for all three tests on Nexus 4 and OnePlus One. For Nexus 4, the results indicate that the Android phone application is the most energy efficient approach, followed by Skype over WLAN. Using Skype over the cellular interface dramatically increases the energy costs of phone calls due to long time to establish a connection. For OnePlus One, calls using Skype over WLAN are the most energy efficient.

Table 3. Time and energy for making a phone call: comparative study (Nexus 4 and OnePlus One)

\begin{tabular}{|l|l|l|l|}
\hline Activity & Time to establish & Total Energy & Energy Overhead \\
\hline
\end{tabular}




\begin{tabular}{|c|c|c|c|c|c|c|}
\hline & \multicolumn{2}{|c|}{ connection [s] } & \multicolumn{2}{c|}{ (ET) [J] } & \multicolumn{2}{c|}{ (OE) [J] } \\
\hline Smartphone & Nexus & OnePlus & Nexus & OnePlus & Nexus & OnePlus \\
\hline $\begin{array}{c}\text { Android Phone over } \\
\text { cellular interface }\end{array}$ & 8.40 & 8.13 & $\underline{33.06}$ & 60.97 & $\underline{11.67}$ & 38.78 \\
\hline $\begin{array}{c}\text { Skype over cellular } \\
\text { interface }\end{array}$ & 34.75 & 10.41 & 88.80 & 57.45 & 51.6 & 34.18 \\
\hline Skype over WLAN & 10.42 & 8.74 & 38.12 & $\underline{42.50}$ & 15.79 & $\underline{18.81}$ \\
\hline
\end{tabular}

Once the connection goes through, the callee awaits $\sim 7 \mathrm{~s}$ for ring time and converses for $\sim 12 \mathrm{~s}$.

\section{Processing and Communication Costs on Smart Button}

Our environment for energy profiling of embedded devicescan be used to provide insights that can help inform the embedded developers on the amount of energy spent by an embedded platform in different operating modes. To illustrate this we profile Smart Button under different operating modes as described in Section IV. We measure the energy for the following operating modes: (a) Smart Button idles waiting for a start command over the Bluetooth interface (b)Smart Button continuously samples inertial sensors with frequency of $100 \mathrm{~Hz}$ and streams samples via its Bluetooth interface for $30 \mathrm{~s}$, and(c) Smart Button performs sampling and on-platformprocessing of sensor signals to detect posture transitions and send extracted parameters at the end of the test via its Bluetooth interface. We would like to determine how these options compare to each other regarding the total energy use.

Table 4 summarizes times and energies for these modes. The mode with on-platform signal processing requires less energy than the mode with streaming raw samples. The on-board signal processing overhead energy is negligible when compared to the idling platform. The reason for this behavior is because both inertial sensors and Bluetooth interface are continually active and their energy expenditure by far exceeds the energy consumed by Teensy on signal processing.

Table 4. Time and energy consumed by Smart Button

\begin{tabular}{|c|c|c|c|c|}
\hline Activity & Mode & $\begin{array}{c}\text { Execution } \\
\text { Time }[\mathbf{s}]\end{array}$ & $\begin{array}{c}\text { Total Energy } \\
\text { (ET) [J] }\end{array}$ & $\begin{array}{c}\text { Energy Overhead } \\
\text { (OE) [J] }\end{array}$ \\
\hline Idle & $\begin{array}{c}\text { Waiting for } \\
\text { commands }\end{array}$ & 30.22 & 9.39 & - \\
\hline
\end{tabular}




\begin{tabular}{|c|c|c|c|c|}
\hline $\begin{array}{c}\text { 30-Second Chair } \\
\text { Stand Test }\end{array}$ & $\begin{array}{c}\text { Sending raw } \\
\text { samples }\end{array}$ & 33.72 & 12.47 & 1.65 \\
\hline $\begin{array}{c}\text { 30-Second Chair } \\
\text { Stand Test }\end{array}$ & $\begin{array}{c}\text { On-board } \\
\text { processing }\end{array}$ & 30.57 & 9.79 & $\sim 0.0$ \\
\hline
\end{tabular}

VI. RELATED WORK

We are aware of several related studies that investigate energy efficiency on mobile devices using custom measurement environments for capturing power traces and logging to capture execution history [4], [5], [25], [10].Rice and Hay [4], [5] evaluated energy efficiency of Android-based G1, Magic, and Hero handsets using their custom measurement setup. Their setup includes a replacement battery and a high-precision shunt resistor placed in series on the power line and an NI data acquisition device that samples voltage drop across the resistor. Their excellent studies focused on measurement-based evaluation and optimization of wireless communication in mobile handsets. Similar setup is used in our prior study focusing on energy-efficiency of Pandaboard and Raspberry Pi development platforms that run Linux operating system [10]. The setup included features to allow automated power measurements for a number of profiled applications. The setup proposed in this paper offers several advantages over the setups introduced in [4], [5], [10]. For example, we utilize Android Debug Bridge (adb) to remotely control the mobile device and launch script command files for unobtrusive power measurements. Next, we use network time synchronization protocol to precisely capture activities on the mobile device and synchronize the current samples collected on the workstation with these activities. Our use of the battery simulator eliminates any voltage changes across the shunt resistor due to drainage of the battery. Additionally, mLViewPowerProfile offers flexible control and automation of experiments.

Study by Shye et al. [25] relies on power models and extended activity logging to generate power schemes which can provide substantial energy saving across entire system, while maintaining user satisfaction. Their study was based on Android G1 running Android 1.0 firmware. They also used a setup based on a shunt 
resistor to capture power traces and a custom logger to generate activity traces. However, their setup offered a limited sampling frequency of only $1 \mathrm{~Hz}$.

All these studies demonstrated the importance of having power measurement setup for analyzing energy consumption on mobile devices. They have also shown how such measurement setup can be used to directly identify energy efficiency improvements. Using high precision power measurement environment and logging capabilities, it is possible to create various power models, power schemes, or simply to be able to analyze and debug power consumption of any given task. Our setup with high sampling frequencies, precise current readings, and time-synchronized operation can analyze shorter and discrete activities on mobile devices to help generate more precise models and power schemes.

\section{CONCLUSIONS}

This paper introduces an environment for automated energy measurements and power profiling of mobile and embedded computing devices. The environment utilizes a National Instruments battery simulator that provides an unobtrusive, high-resolution (down to $1 \mu \mathrm{A}$ ) and high-frequency sampling (down to $5 \mu \mathrm{s}$ ) of the current drawn by a mobile device. Our custom program mLViewPowerProfile running on a workstation interfaces both the device under test and the battery simulator to synchronize the collection of samples from the battery simulator and running applications on the device. mLViewPowerProfile connects to the mobile device over Android debug interface and runs script commands to allow for a full automation of profiling with no user intervention. For embedded computing devices, a data acquisition device is used to synchronizesoftware-initiated hardware triggers and the collection of current samples from the battery simulator.

The paper describes several approaches to profiling Androidand bare-metal embedded applications, which give software developers and researchers an opportunity to gain a deeper insight into application power requirements. Finally, we present a number of case studies that demonstrate capabilities of the proposed setup and its usefulness in increasing energy-efficiency of mobile and wearable devices. 


\section{ACKNOWLEDGMENTS}

This work has been supported in part by National Science Foundation grants CNS-1205439 and CNS1217470.

\section{REFERENCES}

[1] Gartner, Inc., "Worldwide Device Shipments to Grow 1.9 Percent in 2016, While End-User Spending to Decline for the First Time," 2016. [Online]. Available: http://www.gartner.com/newsroom/id/3187134. [Accessed: 13Feb-2016].

[2] IDC, “Apple, Huawei, and Xiaomi Finish 2015 with Above Average Year-Over-Year Growth, as Worldwide Smartphone Shipments Surpass 1.4 Billion for the Year, According to IDC," www.idc.com, 2016. [Online]. Available: http://www.idc.com/getdoc.jsp?containerId=prUS40980416. [Accessed: 13-Feb-2016].

[3] A. Carroll and G. Heiser, "An analysis of power consumption in a smartphone," in Proceedings of the 2010 USENIX conference on USENIX annual technical conference, Berkeley, CA, USA, 2010, pp. 21-21.

[4] A. Rice and S. Hay, "Decomposing power measurements for mobile devices," in 2010 IEEE International Conference on Pervasive Computing and Communications (PerCom), 2010, pp. 70-78.

[5] A. Rice and S. Hay, "Measuring mobile phone energy consumption for 802.11 wireless networking," Pervasive Mob. Comput., vol. 6, no. 6, pp. 593-606, Dec. 2010.

[6] W. L. Bircher and L. K. John, "Complete System Power Estimation Using Processor Performance Events," IEEE Trans. Comput., vol. 61, no. 4, pp. 563-577, Apr. 2012.

[7] A. Pathak, Y. C. Hu, and M. Zhang, "Where is the energy spent inside my app?: fine grained energy accounting on smartphones with Eprof," in Proceedings of the 7th ACM european conference on Computer Systems, New York, NY, USA, 2012, pp. 29-42.

[8] A. Pathak, Y. C. Hu, M. Zhang, P. Bahl, and Y.-M. Wang, "Fine-grained power modeling for smartphones using system call tracing," in Proceedings of the sixth conference on Computer systems, New York, NY, USA, 2011, pp. 153-168.

[9] T. Li and L. K. John, "Run-time modeling and estimation of operating system power consumption," SIGMETRICS Perform Eval Rev, vol. 31, no. 1, pp. 160-171, Jun. 2003.

[10] M. Milosevic, A. Dzhagaryan, E. Jovanov, and A. Milenković, “An Environment for Automated Power Measurements on Mobile Computing Platforms," in Proceedings of the 51st ACM Southeast Conference, New York, NY, USA, 2013, p. 6.

[11] A. Milenkovic, M. Milenkovic, E. Jovanov, D. Hite, and D. Raskovic, "An environment for runtime power monitoring of wireless sensor network platforms," in System Theory, 2005. SSST'05. Proceedings of the ThirtySeventh Southeastern Symposium on, 2005, pp. 406-410.

[12] NI, “NI PXIe-4154 - National Instruments,” 2014. [Online]. Available: http://sine.ni.com/nips/cds/view/p/lang/en/nid/209090. [Accessed: 20-Jun-2014]. 
[13] Google, "Nexus - Google," 2014. [Online]. Available: http://www.google.com/intl/all/nexus. [Accessed: 15-Jun2014].

[14] Google, “Android,” 2014. [Online]. Available: http://www.android.com/. [Accessed: 20-Jun-2014].

[15] OnePlus, “OnePlus One,” 2015. [Online]. Available: https://oneplus.net/one. [Accessed: 12-Jul-2015].

[16] CyanogenMod, "CyanogenMod | Android Community Operating System," 2014. [Online]. Available: http://www.cyanogenmod.org/. [Accessed: 14-Jun-2014].

[17] A. Dzhagaryan, A. Milenkovic, E. Jovanov, and M. Milosevic, "Smart Button: A Wearable System for Assessing Mobility in Elderly," in Proceedings of the 17th International Conference on E-health, Networking, Application \& Services (HealthCom'15), Boston, MA, USA, 2015.

[18] Qualcomm, "Snapdragon ${ }^{\mathrm{TM}}$ Mobile Processors - Qualcomm Developer Network," 2014. [Online]. Available: https://developer.qualcomm.com/discover/chipsets-and-modems/snapdragon. [Accessed: 20-Jun-2014].

[19] Qualcomm, “Adreno,” Qualcomm Developer Network, 2015. [Online]. Available: https://developer.qualcomm.com/software/adreno-gpu-sdk/gpu. [Accessed: 14-Jun-2015].

[20] NI, “NI PXIe-1073 - National Instruments,” 2014. [Online]. Available: http://sine.ni.com/nips/cds/view/p/lang/en/nid/207401. [Accessed: 20-Jun-2014].

[21] Google, “Android Debug Bridge | Android Developers,” 2015. [Online]. Available: http://developer.android.com/tools/help/adb.html. [Accessed: 14-Jun-2015].

[22] Google, "Log | Android Developers," 2014. [Online]. Available: http://developer.android.com/reference/android/util/Log.html. [Accessed: 03-Aug-2014].

[23] A. Milenkovic, A. Dzhagaryan, and M. Burtscher, "Performance and Energy Consumption of Lossless Compression/Decompression Utilities on Mobile Computing Platforms," in 2013 IEEE 21st International Symposium on Modeling, Analysis Simulation of Computer and Telecommunication Systems (MASCOTS), 2013, pp. 254-263.

[24] “Cisco Visual Networking Index: Global Mobile Data Traffic Forecast Update, 2011-2016 [Visual Networking Index (VNI)] - Cisco Systems.” [Online]. Available: http://www.cisco.com/en/US/solutions/collateral/ns341/ns525/ns537/ns705/ns827/white_paper_c11520862.html. [Accessed: 01-Jun-2012].

[25] A. Shye, B. Scholbrock, and G. Memik, "Into the wild: Studying real user activity patterns to guide power optimizations for mobile architectures," in 42nd Annual IEEE/ACM International Symposium on Microarchitecture, 2009. MICRO-42, 2009, pp. 168-178. 


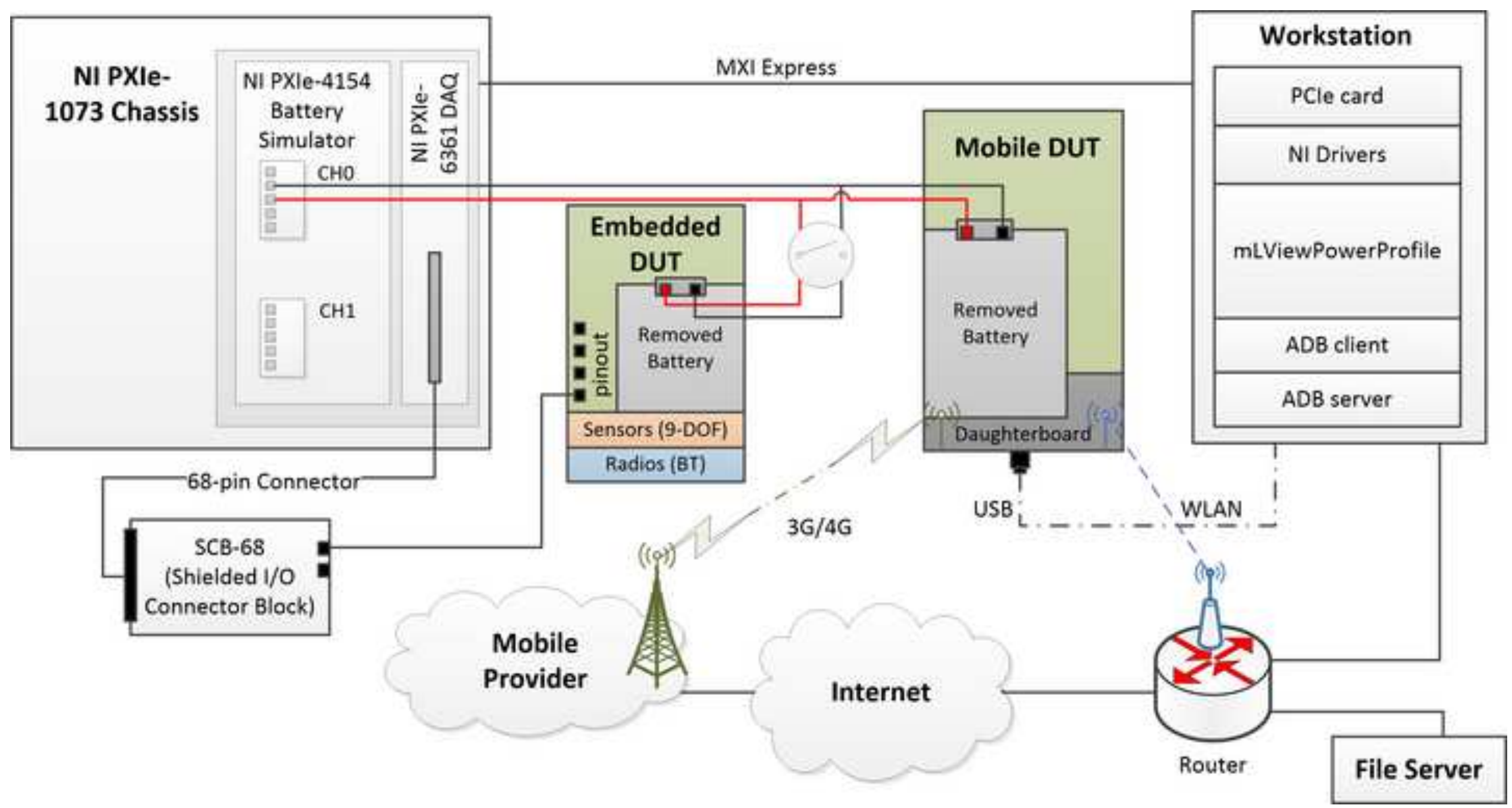

